QUARTERLY OF APPLIED MATHEMATICS

VOLUME LXVI, NUMBER 4

DECEMBER 2008, PAGES 609-632

S 0033-569X(08)01100-0

Article electronically published on September 10, 2008

\title{
SOLID-LIQUID PHASE CHANGES WITH DIFFERENT DENSITIES
}

\author{
BY \\ MICHEL FRÉMOND (Dipartimento di Ingegneria Civile, Università di Roma "Tor Vergata", Via \\ del Politecnico, 1, I-00133 Roma, Italy) \\ AND \\ ELISABETTA ROCCA (Dipartimento di Matematica, Università di Milano, Via Saldini, 50, \\ I-20133 Milano, Italy)
}

\begin{abstract}
In this paper we present a new thermodynamically consistent phase transition model describing the evolution of a liquid substance, e.g., water, in a rigid container $\Omega$ when we freeze the container. Since the density $\varrho_{2}$ of ice with volume fraction $\beta_{2}$ is lower than the density $\varrho_{1}$ of water with volume fraction $\beta_{1}$, experiments, for instance the freezing of a glass bottle filled with water, show that the water pressure increases up to the rupture of the bottle. When the container is not impermeable, freezing may produce a nonhomogeneous material, for instance water, ice or sorbet. Here we describe a general class of phase transition processes, including this example as a particular case. Moreover, we study the resulting nonlinear and singular PDE system from the analytical viewpoint, recovering existence of a global (in time) weak solution and also uniqueness for some particular choices of the nonlinear functions involved.
\end{abstract}

1. Introduction. In this paper we consider a phase change process occurring in a bounded container $\Omega$ of $\mathbb{R}^{3}$. More in particular, we consider a liquid substance (with density $\varrho_{1}$ ) in a rigid and impermeable container $\Omega$ and freeze it. We assume that the solid phase has zero velocity, while the liquid phase velocity is the vector $\mathbf{U}_{1}$, let $\beta_{1}$ be the local proportion of the liquid phase, $\beta_{2}$ the local proportion of the solid phase (with density $\varrho_{2}<\varrho_{1}$ ), and $\vartheta$ the absolute temperature of the system. In this setting (cf. the following Section 2 for further explanations on the model), it is possible to observe macroscopically the formation of voids; thus we do not have the condition $\beta_{1}+\beta_{2}=1$, but we only know that $\beta_{1}+\beta_{2} \in[0,1]$. In the following Section 2 we introduce the model which will lead to the following PDE system we study, coupling it with suitable

Received February 26, 2007.

2000 Mathematics Subject Classification. Primary 80A22, 34A34, 74G25.

Key words and phrases. Phase transitions with voids, singular and nonlinear PDE system, global existence of solutions.

Current address: CMLA, ENS Cachan -Département de Mécanique, ENSTA, Paris.

E-mail address: fremond@cmla.ens-cachan.fr

E-mail address: rocca@mat.unimi.it

(C)2008 Brown University 
initial and boundary conditions, from the analytical viewpoint in the next Sections 35 . We search for solutions $\left(\vartheta, \beta_{1}, \beta_{2}, p\right)$ ( $p$ standing for the pressure) of the Cauchy problem associated with the following PDE system, coupled with suitable boundary conditions, in the cylindrical domain $Q_{T}:=\Omega \times(0, T), T$ being the final time of the process:

$$
\begin{aligned}
& k \partial_{t} p+\varrho_{1} \partial_{t} \beta_{1}+\varrho_{2} \partial_{t} \beta_{2}-\Delta p=0, \\
& \partial_{t}(\log \vartheta)+\frac{\ell}{\vartheta_{c}} \partial_{t} \beta_{1}-\Delta(\log \vartheta)=R, \\
& \left(\begin{array}{c}
\partial_{t} \beta_{1} \\
\partial_{t} \beta_{2}
\end{array}\right)-\nu\left(\begin{array}{c}
\Delta \beta_{1} \\
\Delta \beta_{2}
\end{array}\right)-p\left(\begin{array}{l}
\varrho_{1} \\
\varrho_{2}
\end{array}\right)+\left(\begin{array}{c}
-\frac{\ell}{\vartheta_{c}}\left(\vartheta-\vartheta_{c}\right) \\
0
\end{array}\right)+\partial \varphi\left(\beta_{1}, \beta_{2}\right) \ni 0 .
\end{aligned}
$$

Here $\Delta$ represents the Laplacian with respect to the space variables and we have assumed that $k, \ell$ and $\vartheta_{c}$ are three constants denoting the compressibility of the substance, the latent heat and the critical temperature of the phase transition process, respectively, while $R$ stands for an entropy source. Moreover, $\nu$ represents a nonnegative interfacial energy coefficient and $\varphi$ is a general proper, convex and lower-semicontinuous function, taking possibly value $+\infty$ in some points. Hence, $\partial \varphi$ represents here the subdifferential of Convex Analysis (cf., e.g., 20]) and it takes possibly into account the restrictions on the phase field variables $\beta_{1}$ and $\beta_{2}$. Indeed, it may be the indicator function of the set of admissible values of $\beta_{1}$ and $\beta_{2}$. If $K=\left\{\left(\beta_{1}, \beta_{2}\right) \in[0,1] \mid \beta_{1}+\beta_{2} \in[0,1]\right\}$, then a possible choice in our analysis is $\varphi\left(\beta_{1}, \beta_{2}\right)=I_{K}\left(\beta_{1}, \beta_{2}\right)$, which is defined to be 0 if $\left(\beta_{1}, \beta_{2}\right) \in K$ and $+\infty$ otherwise. This is the reason why we have an inclusion symbol in (1.3), since the $\partial \varphi$ may possibly be a multivalued operator.

We refer to Section 2 for the detailed description of our model, but we prefer to anticipate that description with a few words here on the derivation of equations (1.1)(1.3). Equation (1.1) follows from the mass balance (cf. also [16]) and the equation of macroscopic motion, which is here assumed to be in agreement with the standard Darcy law (cf. 14]). Equation (1.2) represents the rescaled internal energy balance, which is a consequence of a new approach developed in [6] (and studied also in [3])-[5], [7]- 9], and [16]). Note that the presence of the logarithmic contribution in (1.2) introduces new difficulties in the mathematical analysis of the problem, due to its singular character, but, once one has proved that a solution exists in some proper sense, this immediately entails the positivity of the temperature, which could otherwise represent a difficult step in the proof (cf., e.g., [13]). Inclusion (1.3) stands for the microscopic motion equation (cf. 15] and also [11] and 22] for different approaches) for a proper choice of the free-energy functional and of the pseudo-potential of dissipation which, following the theory of [20, accounts for dissipation in the model (cf. also the next Section 2 for further details on this topic).

In all the references quoted above (except for [16]) only phase transition processes in which no voids nor overlapping between phases can occur are studied. In that case the proofs mainly rely on the fact that $\beta_{1}+\beta_{2}=1$; hence the mass balance equation as well as the effects of the pressure can be neglected and so the system reduces to the coupling of equation (1.2) (with only one phase variable, say $\chi=\beta_{1}-\beta_{2}$ ) and (1.3). More in particular, in [6] the model coupling these two equations and accounting 
also for some memory effects in (1.2) is studied and an existence (of a weak solution) result is proved in case $\varphi=I_{[0,1]}$, while uniqueness still remains an open problem. In [7] and [4, 5] the uniqueness of a solution is established and also some investigation on the long-time behaviour of solutions is performed respectively in case $\nu=0$ and without any thermal memory for a system in which a different heat flux law in (1.2) (leading to an internal energy balance with $\vartheta$ instead of $\log \vartheta$ inside the Laplacian) is taken into account. Moreover, in [9] (and in [3] for the case $\nu=0$ and without any thermal memory contribution) the long-time behaviour of the trajectories of the solutions to an extension of the model presented in [6] is studied, while in 8 8 a more general approach to these kinds of processes (leading to a more general type of nonlinearities in the equations) is performed. In particular in [8] the $\log \vartheta$ term in (1.2) is substituted by a more general nonlinear function (maybe also multivalued) including the logarithmic function as a particular case. An existence-long-time behaviour of solution result is established in [8]. Only in [16], instead, is a first attempt developed to approach phasefield systems including the possibility of having voids. In [16] indeed we study a system coupling the phase equation (1.3) (including also a viscosity term), the temperature equation (1.2), a general mass balance equation, and a quasi-static standard stress-strain relation, including viscosity effects but no accelerations. We establish in [16] a global well-posedness result for the resulting PDE system coupled with suitable initial and boundary conditions.

Here, under the assumption that the solid phase velocity is 0 and by means of the Darcy law (cf. 14 and also (2.16) below), the mass balance equation can be rewritten as (3.15). The main mathematical difficulties stand in the nonlinear and singular coupling between (3.16) and (3.17). Our approach to this system is to regularize the log term in (1.2) by introducing its Lipschitz-continuous approximation. Hence, we first solve the regularized problem in Section 4 getting also uniqueness of solutions; then, in the last Section 5 we prove our main result (stated in Section 3) entailing global (in time) existence of (weak) solutions to (1.1)-(1.3) coupled with suitable initial and boundary conditions. The problem of uniqueness for the system (1.1)-(1.3) is still open (cf. also Remark 3.8 below) and this is mainly due to the lack of regularity of the $\vartheta$-component of the solution $\left(\vartheta, \beta_{1}, \beta_{2}, p\right)$ to (1.1)-(1.3).

2. The model. In this section we detail the derivation of the model leading to the PDE system (1.1)-(1.3) already introduced in Section 1. Hence, let us consider a liquid substance, e.g., water, in a rigid and impermeable container $\Omega$ and freeze the container. Since the density $\varrho_{2}$ of ice with volume fraction $\beta_{2}$ is lower than the density $\varrho_{1}$ of water with volume fraction $\beta_{1}$, it seems impossible that water transforms into ice (or, more in general, into a mixture of ice and water): the water remains liquid even at low temperature. Experiments, for instance the freezing of a glass bottle filled with water, show that the water pressure increases up to the rupture of the bottle. When the container is not impermeable, freezing may produce a nonhomogeneous material, for instance, water, ice or sorbet. In this setting, it is possible to observe macroscopically the presence of voids and thus we do not have the condition $\beta_{1}+\beta_{2}=1$, but we can only deduce that $0 \leq \beta_{1}+\beta_{2} \leq 1$. 
We assume the ice has zero velocity, while the water velocity is represented by the vector $\mathbf{U}_{1}$. For the sake of simplicity, we assume the small perturbation assumption (cf. [17). In this setting the mass balance is

$$
\begin{aligned}
& \beta_{1} \partial_{t} \varrho_{1}+\beta_{2} \partial_{t} \varrho_{2}+\varrho_{1} \partial_{t} \beta_{1}+\varrho_{2} \partial_{t} \beta_{2}+\varrho_{1} \beta_{1} \operatorname{div} \mathbf{U}_{1}=0, \\
& -\varrho_{1} \beta_{1} \mathbf{U}_{1} \cdot \mathbf{n}=\varpi,
\end{aligned}
$$

where $\varpi$ is the liquid water intake and by $\mathbf{n}$ we denote the outward unitary normal vector to $\partial \Omega$. We have assumed the substance to be compressible, although in some cases this compressibility is very low. Moreover, the equation of motion for the macroscopic motion of the water can be written as

$$
\begin{aligned}
& \varrho_{1} \partial_{t}\left(\beta_{1} \mathbf{U}_{1}\right)=\operatorname{div} \sigma_{1}+\mathbf{f}_{1}, \\
& \sigma_{1} \mathbf{n}=\mathbf{g}_{1},
\end{aligned}
$$

where $\sigma_{1}$ is the water stress, $\mathbf{f}_{1}$ is the action of the exterior on the liquid water, for instance the friction of the water on the solid ice phase, and $\mathbf{g}_{1}$ is the boundary exterior action on the liquid water.

For the microscopic forces responsible for the ice water phase change, the equation of motion is (cf. [15])

$$
-\left(\begin{array}{c}
B_{1} \\
B_{2}
\end{array}\right)+\operatorname{div}\left(\begin{array}{l}
\mathbf{H}_{1} \\
\mathbf{H}_{2}
\end{array}\right)=0
$$

This vector equation is the equation of microscopic motion and it deals with the works produced by the microscopic motions occurring in the phase changes. The terms $B_{1}$, $B_{2}, \mathbf{H}_{1}$, and $\mathbf{H}_{2}$ are new internal forces responsible for phase transition phenomena. In particular $B_{1}$ represents the energy density per unit of $\beta_{1}, B_{2}$ represents the energy density per unit of $\beta_{2}$, while $H_{1}$ and $H_{2}$ represent densities of energy flux. If we assume that no exterior source of work is present, we also have

$$
\left(\begin{array}{l}
\mathbf{H}_{1} \\
\mathbf{H}_{2}
\end{array}\right) \cdot \mathbf{n}=0
$$

The internal energy balance reformulated in terms of the entropy $s$ is (cf. [6] for its derivation)

$$
\partial_{t} s+\operatorname{div} \mathbf{Q}=R,
$$

where we have denoted by $\mathbf{Q}$ the entropy flux, by $R$ the entropy source, $r=R \vartheta$ being the heat source, and by $\boldsymbol{\beta}$ the column vector of components $\beta_{1}, \beta_{2}$. No high order dissipative contribution is involved on the right-hand side due to the small perturbation assumption.

The internal forces are split into dissipative forces indexed by ${ }^{d}$ and nondissipative interior forces indexed by ${ }^{n d}$. The nondissipative forces are defined through the water free energy $\Psi$, which takes into account the internal constraint on the state quantities, for instance

$$
\left(\beta_{1}, \beta_{2}\right) \in K=\left\{\left(\gamma_{1}, \gamma_{2}\right) \in[0,1] \mid \gamma_{1}+\gamma_{2} \in[0,1]\right\}
$$


We choose the following form for the free energy:

$$
\begin{aligned}
\Psi\left(\vartheta, \varrho_{1}, \varrho_{2}, \beta_{1}, \beta_{2}, \nabla \beta_{1}, \nabla \beta_{2}\right)= & -c_{0} \vartheta(\log \vartheta-1)-\frac{\beta_{1} \ell}{\vartheta_{c}}\left(\vartheta-\vartheta_{c}\right)+I_{K}\left(\beta_{1}, \beta_{2}\right) \\
& +\frac{\nu}{2}\left(\left|\nabla \beta_{1}\right|^{2}+\left|\nabla \beta_{2}\right|^{2}\right)+\beta_{1} f_{1}\left(\varrho_{1}\right)+\beta_{2} f_{2}\left(\varrho_{2}\right),
\end{aligned}
$$

where $\vartheta_{c}$ is the critical temperature of the system, $\ell$ denotes the latent heat of the melting solidification process, $c_{0}$ the positive specific heat, $\nu$ a positive interfacial energy coefficient, and $I_{K}$ stands for the indicator function of the set $K$ (it takes the value 0 on $K$ and $+\infty$ otherwise). Because we have assumed the substance to be compressible, the free energy depends on the densities. Indeed, $f_{1}$ and $f_{2}$ account, respectively, for the dependence on the densities of the free energy related to the liquid and the solid phases. Thus we have

$$
s=-\frac{\partial \Psi}{\partial \vartheta}=c_{0} \log \vartheta+\frac{\ell \beta_{1}}{\vartheta_{c}}
$$

and we define the nondissipative internal forces as:

$$
\begin{aligned}
& \left(\begin{array}{c}
B_{1}^{n d} \\
B_{2}^{n d}
\end{array}\right)=\left(\begin{array}{c}
\frac{\partial \Psi}{\partial \beta_{1}} \\
\frac{\partial \Psi}{\partial \beta_{2}}
\end{array}\right) \in\left(\begin{array}{c}
-\frac{\ell}{\vartheta_{c}}\left(\vartheta-\vartheta_{c}\right) \\
0
\end{array}\right)+\partial I_{K}\left(\beta_{1}, \beta_{2}\right), \\
& \left(\begin{array}{c}
\mathbf{H}_{1}^{n d} \\
\mathbf{H}_{2}^{n d}
\end{array}\right)=\left(\begin{array}{c}
\frac{\partial \Psi}{\partial\left(\nabla \beta_{1}\right)} \\
\frac{\partial \Psi}{\partial\left(\nabla \beta_{2}\right)}
\end{array}\right)=\nu\left(\begin{array}{c}
\nabla \beta_{1} \\
\nabla \beta_{2}
\end{array}\right), \\
& \left(\begin{array}{c}
P_{1}^{n d} \\
P_{2}^{n d}
\end{array}\right)=\left(\begin{array}{c}
\frac{\partial \Psi}{\partial \varrho_{1}} \\
\frac{\partial \Psi}{\partial \varrho_{2}}
\end{array}\right)=\left(\begin{array}{c}
\beta_{1} \frac{\partial f_{1}}{\partial \varrho_{1}} \\
\beta_{2} \frac{\partial f_{2}}{\partial \varrho_{2}}
\end{array}\right),
\end{aligned}
$$

with an abuse of notation for the derivatives $\partial \Psi / \partial \beta$ and where $\partial I_{K}$ represents the subdifferential of $I_{K}$ in the sense of Convex Analysis (cf., e.g., [10] or [20]). Note that we can get rid in (2.7) of the vector $\left(f_{1}\left(\varrho_{1}\right), f_{2}\left(\varrho_{2}\right)\right.$ ) (which should result from $\partial \psi / \partial \beta$ ) by means of the small perturbations assumption (cf. (2.17)-(2.18). Because the free energy does not depend on deformations, we have

$$
\sigma_{1}^{n d}=0 .
$$

The dissipative forces are defined with the pseudopotential of dissipation $\Phi$, which takes into account the internal constraints related to the velocities and ensures that the second law of thermodynamics is satisfied. The mass balance represents such a constraint (cf. also [16] for further comments on this choice). For instance, we may choose

$$
\begin{aligned}
\Phi\left(\partial_{t} \varrho_{1}, \partial_{t} \varrho_{2}, \partial_{t} \beta_{1}, \partial_{t} \beta_{2},\right. & \left.\nabla\left(\partial_{t} \beta_{1}\right), \nabla\left(\partial_{t} \beta_{2}\right), D\left(\mathbf{U}_{1}\right), \nabla \vartheta\right) \\
= & I_{0}\left(\beta_{1} \partial_{t} \varrho_{1}+\beta_{2} \partial_{t} \varrho_{2}+\varrho_{1} \partial_{t} \beta_{1}+\varrho_{2} \partial_{t} \beta_{2}+\varrho_{1} \beta_{1} \operatorname{div} \mathbf{U}_{1}\right) \\
& +\frac{\mu}{2}\left(\left|\partial_{t} \beta_{1}\right|^{2}+\left|\partial_{t} \beta_{2}\right|^{2}\right)+\frac{\lambda}{2 \vartheta}|\nabla \vartheta|^{2},
\end{aligned}
$$


where $I_{0}$ denotes the indicator function of the origin of $\mathbb{R}, \mu$ the phase change viscosity and $\lambda>0$ denotes the heat conductivity of the system, while the operator $D$ represents the linearized symmetric strain tensor $D_{i j}(\mathbf{U}):=\left(U_{i, j}+U_{j, i}\right) / 2$, where $i, j=1,2,3$ and with the commas we mean space derivatives. The dissipative forces can be written as:

$$
\begin{aligned}
\left(P_{1}^{d}, P_{2}^{d}, B_{1}^{d}, B_{2}^{d}, \mathbf{H}_{1}^{d},\right. & \left.\mathbf{H}_{2}^{d}, \sigma_{1}^{d}, \mathbf{Q}^{d}\right) \\
& \in \partial \Phi\left(\partial_{t} \varrho_{1}, \partial_{t} \varrho_{2}, \partial_{t} \beta_{1}, \partial_{t} \beta_{2}, \nabla\left(\partial_{t} \beta_{1}\right), \nabla\left(\partial_{t} \beta_{2}\right), D\left(\mathbf{U}_{1}\right), \nabla \vartheta\right),
\end{aligned}
$$

where $\partial \Phi$ represents the subdifferential of $\Phi$, and the result is

$$
\begin{aligned}
& \mathbf{Q}=\mathbf{Q}^{d}=-\frac{\partial \Phi}{\partial \nabla \vartheta}=-\lambda \nabla(\log \vartheta), \\
& \mathbf{H}_{1}^{d}=\frac{\partial \Phi}{\partial\left(\nabla \partial_{t} \beta_{1}\right)}=0, \mathbf{H}_{2}^{d}=\frac{\partial \Phi}{\partial\left(\nabla \partial_{t} \beta_{2}\right)}=0, \\
& \left(\begin{array}{c}
P_{1}^{d} \\
P_{2}^{d} \\
B_{1}^{d} \\
B_{2}^{d} \\
\sigma_{1}^{d}
\end{array}\right)=\left(\begin{array}{c}
\frac{\partial \Phi}{\partial\left(\partial_{t} \varrho_{1}\right)} \\
\frac{\partial \Phi}{\partial\left(\partial_{t} \varrho_{2}\right)} \\
\frac{\partial \Phi}{\partial\left(\partial_{t} \beta_{1}\right)} \\
\frac{\partial \Phi}{\partial\left(\partial_{t} \beta_{2}\right)} \\
\frac{\partial \Phi}{\partial D\left(\mathbf{U}_{1}\right)}
\end{array}\right) \in\left(\begin{array}{c}
0 \\
0 \\
\mu \partial_{t} \beta_{1} \\
\mu \partial_{t} \beta_{2} \\
0
\end{array}\right)+\partial I_{0}(0)\left(\begin{array}{c}
\beta_{1} \\
\beta_{2} \\
\varrho_{1} \\
\varrho_{2} \\
\varrho_{1} \beta_{1} \mathbf{1}
\end{array}\right),
\end{aligned}
$$

with an abuse of notation for the derivatives $\partial \Phi / \partial\left(\partial_{t} X\right)$ and where $\mathbf{1}$ stands for the unit tensor. By denoting $-p$ for an element of $\partial I_{0}$, the last relations can be written as:

$$
\begin{aligned}
& P_{1}^{d}=-p \beta_{1}, P_{2}^{d}=-p \beta_{2}, \\
& B_{1}^{d}=\mu \partial_{t} \beta_{1}-p \varrho_{1}, B_{2}^{d}=\mu \partial_{t} \beta_{2}-p \varrho_{2}, \\
& \sigma_{1}^{d}=-p \varrho_{1} \beta_{1} \mathbf{1} .
\end{aligned}
$$

The quantity $p \varrho_{1} \beta_{1}$ turns out to be the pressure in the liquid phase and using the constitutive law (2.15) in the equation for the macroscopic motion (2.2) we get

$$
\varrho_{1} \partial_{t}\left(\beta_{1} \mathbf{U}_{1}\right)=-\nabla\left(p \varrho_{1} \beta_{1}\right)+\mathbf{f}_{1} .
$$

We may assume that the acceleration is negligible and that the volume exterior force $\mathbf{f}_{1}$ results mainly from friction on the solid phase and that this force is proportional to the relative velocity through the relation

$$
\mathbf{f}_{1}=-\frac{\left(\varrho_{1} \beta_{1}\right)^{2}}{m} \mathbf{U}_{1} .
$$

This is mainly due to the fact that the force has to be $\mathbf{0}$ when there is no water.

REMARK 2.1. The density of the force $\mathbf{f}_{1}$ with respect to the actual volume of water $\beta_{1} d \Omega$ is

$$
\frac{\mathbf{f}_{1}}{\beta_{1}}=-\frac{\varrho_{1}^{2} \beta_{1}}{m} \mathbf{U}_{1} .
$$

It is proportional to $\beta_{1}$ and to the liquid water momentum $\varrho_{1} \beta_{1} \mathbf{U}_{1}$ as one may expect. 
With this choice, from (2.2) we get

$$
\frac{\left(\varrho_{1} \beta_{1}\right)^{2}}{m} \mathbf{U}_{1}=-\nabla\left(p \varrho_{1} \beta_{1}\right),
$$

which (within the small perturbation theory (cf. [17])) can be rewritten as:

$$
\varrho_{1} \beta_{1} \mathbf{U}_{1}=-m \nabla p
$$

where the parameter $m$ denotes the mobility of the water in agreement with the Darcy law (cf. [14]).

Since the densities $\varrho_{1}$ and $\varrho_{2}$ are not related to a particular internal force, their constitutive laws are (cf. [15, (3.25), p. 11])

$$
P_{1}^{n d}+P_{1}^{d}=0, \quad P_{2}^{n d}+P_{2}^{d}=0,
$$

which, with (2.9) and (2.13), can be written as:

$$
\frac{\partial f_{1}}{\partial \varrho_{1}}-p=0, \quad \frac{\partial f_{2}}{\partial \varrho_{2}}-p=0 .
$$

Within the small perturbation assumption, we have

$$
p=k_{1}\left(\varrho_{1}-\varrho_{1}^{r}\right), \quad p=k_{2}\left(\varrho_{2}-\varrho_{2}^{r}\right),
$$

where the $k_{i}(i=1,2)$ are the compressibilities of the liquid and solid phases, respectively, and the $\varrho_{i}^{r}(i=1,2)$ are reference densities. Let us note that the densities, which depend on the pressure $p$ through (2.18), may also depend on the temperature via a thermal expansion coefficient, giving $p=k_{i}\left(\varrho_{i}-\varrho_{i}^{r}\right)+k_{i}^{t h}\left(\vartheta-\vartheta_{c}\right)$ in place of (2.18). However, for the time being, we do not introduce this dependence.

The predictive theory equations within the small perturbation assumption result from the mass balance (2.1) with (2.18), the equation of motion (2.2)-(2.3), the entropy balance (2.4) and the constitutive laws (2.6) -(2.14) with relation (2.16). Hence, they can be written as follows:

$$
\begin{aligned}
& k \partial_{t} p+\varrho_{1} \partial_{t} \beta_{1}+\varrho_{2} \partial_{t} \beta_{2}-m \Delta p=0 \\
& c_{0} \partial_{t}(\log \vartheta)+\frac{\ell}{\vartheta_{c}} \partial_{t} \beta_{1}-\lambda \Delta(\log \vartheta)=R \\
& \mu\left(\begin{array}{c}
\partial_{t} \beta_{1} \\
\partial_{t} \beta_{2}
\end{array}\right)-\nu\left(\begin{array}{c}
\Delta \beta_{1} \\
\Delta \beta_{2}
\end{array}\right)-p\left(\begin{array}{l}
\varrho_{1} \\
\varrho_{2}
\end{array}\right)+\left(\begin{array}{c}
-\frac{\ell}{\vartheta_{c}}\left(\vartheta-\vartheta_{c}\right) \\
0
\end{array}\right)+\partial I_{K}\left(\beta_{1}, \beta_{2}\right) \ni 0,
\end{aligned}
$$

where $k=\left(\frac{\beta_{1}^{0}}{k_{1}}+\frac{\beta_{2}^{0}}{k_{2}}\right)$ is the compressibility of the mixture, $\beta_{1}^{0}+\beta_{2}^{0}$ being the reference value of the material volume fraction. This set of equations is completed by the following boundary conditions on $\partial \Omega$ :

$$
\begin{aligned}
& \frac{\partial \beta_{1}}{\partial \mathbf{n}}=0, \frac{\partial \beta_{1}}{\partial \mathbf{n}}=0, \\
& \lambda \frac{\partial \vartheta}{\partial \mathbf{n}}+\alpha_{\vartheta}\left(\vartheta-\vartheta_{e x t}\right)=0,
\end{aligned}
$$


where $\alpha_{\vartheta}$ is the thermal conductivity of the boundary of the container. Concerning the hydraulic boundary conditions, we may choose

$$
m \frac{\partial p}{\partial \mathbf{n}}+\alpha_{p}\left(p-p_{e x t}\right)=0,
$$

where $\alpha_{p}=0$ if the boundary is water tight, $\alpha_{p}=\infty$ if the boundary is connected to a water supply with pressure $p_{\text {ext }}$ (in this case the boundary condition is $p=p_{\text {ext }}$ ). This condition means that the flow is proportional to the difference of pressure $p-p_{\text {ext }}$, in case the container is permeable. In the case, instead, of a metallic recipient, the condition accounts for the deformation of the metal due to the pressure.

REMARK 2.2. Due to the small perturbation assumption, the densities $\varrho_{1}$ and $\varrho_{2}$ in equations (2.19) and (2.21) are constant.

REMARK 2.3. More sophisticated boundary conditions result from more sophisticated physical boundary properties. The boundary of the container may be semi-permeable; in this case we have

$$
m \frac{\partial p}{\partial \mathbf{n}}-\alpha_{p}\left(p-p_{\text {ext }}\right)^{-}=0,
$$

where $\left(p-p_{\text {ext }}\right)^{-}=\sup \left(0,-\left(p-p_{\text {ext }}\right)\right)$ is the negative part of $p-p_{\text {ext }}$. This boundary condition means that when the pressure is lower than the exterior pressure $p_{\text {ext }}$, water flows inside the container but when the pressure is larger than the exterior pressure, no water flows outside.

The pressure may also be controlled on the boundary by the following relation:

$$
m \frac{\partial p}{\partial \mathbf{n}}+\partial I_{-}\left(p-p_{\text {ext }}\right) \ni 0 .
$$

Water flows outside the container in order to maintain the pressure lower than the outside pressure $p_{\text {ext }}$, which may be the atmospheric pressure.

The initial conditions in $\Omega$ are

$$
\beta_{1}(x, 0)=\beta_{1}^{0}(x), \beta_{2}(x, 0)=\beta_{2}^{0}(x), \vartheta(x, 0)=\vartheta_{0}(x), p(x, 0)=p_{0}(x) .
$$

This set of partial differential equations allows us to compute the pressure $p(x, t)$, liquid water and ice volume fractions $\beta_{1}(x, t), \beta_{2}(x, t)$ and temperature $\vartheta(x, t)$ depending on the external actions resulting from the exterior pressure $p_{\text {ext }}(x, t)$ and exterior temperature $\vartheta_{\text {ext }}(x, t)$ and rate of heat production $\vartheta_{c} R(x, t)$, which is very often equal to 0 . In an engineering situation indeed the governing action is the exterior temperature cooling and heating the container.

2.1. Some examples.

1. Freezing water in an impermeable container. Suppose $\beta_{1}^{0}=1, \beta_{2}^{0}=0, \vartheta_{0}>0$ and that the boundary condition for the pressure is (2.24) with $\alpha_{p}=0$; i.e.,

$$
\frac{\partial p}{\partial \mathbf{n}}=0 .
$$

Hence, the container is completely cooled. In order to look for closed form solutions we assume all the quantities are homogeneous. Assuming the temperature to be known, we 
focus on equation (2.21), which describes the phase change. Because of homogeneity, it becomes

$$
\mu\left(\begin{array}{c}
\partial_{t} \beta_{1} \\
\partial_{t} \beta_{2}
\end{array}\right)-p\left(\begin{array}{c}
\varrho_{1} \\
\varrho_{2}
\end{array}\right)+\left(\begin{array}{c}
-\frac{\ell}{\vartheta_{c}}\left(\vartheta-\vartheta_{c}\right) \\
0
\end{array}\right)+\partial I_{K}\left(\beta_{1}, \beta_{2}\right) \ni 0 .
$$

When the Celsius temperature $\vartheta-\vartheta_{c}$ is positive, the solution of this equation is $\beta_{1}=1$ and $\beta_{2}=0$. When the Celsius temperature becomes negative, the expected phase change does not occur. Indeed due to the mass balance, to values $\beta_{1}=1$ and $\beta_{2}=0$ and to the condition $\beta_{1}+\beta_{2} \leq 1$, we should have, neglecting the effect of the compressibility, the following relations:

$$
\varrho_{2} \partial_{t} \beta_{2}+\varrho_{1} \partial_{t} \beta_{1}=0, \quad \partial_{t} \beta_{1}<0, \quad \partial_{t} \beta_{2}+\partial_{t} \beta_{1} \leq 0 .
$$

Note that the first two conditions imply

$$
\partial_{t} \beta_{2}+\partial_{t} \beta_{1}=\left(1-\frac{\varrho_{1}}{\varrho_{2}}\right) \partial_{t} \beta_{1}>0
$$

which contradicts the third condition. Let us check that the water remains liquid $\left(\partial_{t} \beta_{1}=\right.$ 0 ) even if the Celsius temperature is negative. We must have

$$
-p\left(\begin{array}{l}
\varrho_{1} \\
\varrho_{2}
\end{array}\right)+\left(\begin{array}{c}
-\frac{\ell}{\vartheta_{c}}\left(\vartheta-\vartheta_{c}\right) \\
0
\end{array}\right)+\partial I_{K}(1,0) \ni 0,
$$

with

$$
\partial I_{K}(1,0)=\left(\begin{array}{c}
P \\
Q
\end{array}\right), P \geq 0, Q \leq P
$$

or

$$
\begin{gathered}
p \varrho_{1}+\frac{\ell}{\vartheta_{c}}\left(\vartheta-\vartheta_{c}\right) \geq 0, \\
0 \leq p\left(\varrho_{1}-\varrho_{2}\right)+\frac{\ell}{\vartheta_{c}}\left(\vartheta-\vartheta_{c}\right) .
\end{gathered}
$$

Since $\varrho_{1}-\varrho_{2}>0$, these conditions may be satisfied. In this case, the minimum value of the pressure is

$$
p=-\frac{\ell}{\left(\varrho_{1}-\varrho_{2}\right) \vartheta_{c}}\left(\vartheta-\vartheta_{c}\right) .
$$

Thus, when cooling the container, the water remains liquid and the pressure increases. This is in agreement with experiment: a glass container filled with water explodes due to the pressure increase when freezing.

2. Freezing of a water emulsion in an impermeable container. Suppose $0<\beta_{1}^{0}<1, \beta_{2}^{0}=$ $0, \vartheta_{0}>0$; hence, the emulsion (a mixture of voids and water) is cooled. Assuming a homogeneous evolution, it may be seen that the water freezes until either $\beta_{1}=0$ (in this case $\beta_{1}^{0}<\varrho_{2} / \varrho_{1}$ and the cooling results in a mixture of ice and voids), or $\beta_{1}+\beta_{2}=1$ (in this case $\beta_{1}^{0} \geq \varrho_{2} / \varrho_{1}$ and the cooling results in a mixture of ice and liquid water, $\left.\beta_{1}=\left(\varrho_{1} \beta_{1}^{0}-\varrho_{2}\right) /\left(\varrho_{1}-\varrho_{2}\right), \beta_{2}=\left(\varrho_{1}-\varrho_{1} \beta_{1}^{0}\right) /\left(\varrho_{1}-\varrho_{2}\right)\right)$. This mixture is water, ice or sorbet. 
3. The ice in a glacier. Assume we have a homogeneous mixture of ice and water without voids at an equilibrium,

$$
\beta_{1}+\beta_{2}=1, \partial_{t} \beta_{1}=\partial_{t} \beta_{2}=0 .
$$

Then equations (2.19) $-(2.21)$ give

$$
-p\left(\begin{array}{l}
\varrho_{1} \\
\varrho_{2}
\end{array}\right)+\left(\begin{array}{c}
-\frac{\ell}{\vartheta_{c}}\left(\vartheta-\vartheta_{c}\right) \\
0
\end{array}\right)+\left(\begin{array}{c}
P \\
P
\end{array}\right)=0
$$

with $P \geq 0$. Hence, we get

$$
-p\left(\varrho_{1}-\varrho_{2}\right)-\frac{\ell}{\vartheta_{c}}\left(\vartheta-\vartheta_{c}\right)=0, p \geq 0 .
$$

In this situation the phase change temperature depends on the pressure. It is known that this is the case for the ice-water phase change. Because $\varrho_{1}>\varrho_{2}$ the phase change temperature decreases when the pressure increases. A consequence of this property is that the ice melts at the bottom of a glacier and lubricates the rock-ice contact surface allowing the downhill motion of the glacier.

3. Variational formulation and main results. In the following part of the paper we want to deal with a suitable generalization of the PDE system (2.19)-(2.21) introduced in the previous Section 2. Hence, we first detail in this section the assumptions on the data and on the general proper, convex, and lower-semicontinuous mapping $\varphi: \mathbb{R}^{2} \rightarrow[0,+\infty]$, which will generalize the role played by the indicator function $I_{K}$ in the inclusion (2.21) introduced in the previous Section 2 , and then we state a suitable variational formulation of the generalized PDE system obtained and our main results.

In order to do that, we first introduce the Hilbert triplet $\left(V, H, V^{\prime}\right)$, where

$$
H:=L^{2}(\Omega) \text { and } V:=H^{1}(\Omega),
$$

and $\Omega \subset \mathbb{R}^{3}$ is a bounded and connected domain with Lipschitz continuous boundary $\Gamma:=\partial \Omega$. Let $T$ be a positive final time of the process and denote by $Q_{t}:=\Omega \times(0, t)$ and $\Sigma_{t}:=\Gamma \times(0, t), t \in(0, T]$. Then, we identify, as usual, $H$ (which stands either for the space $L^{2}(\Omega)$ or for $\left(L^{2}(\Omega)\right)^{3}$ or for $\left.\left(L^{2}(\Omega) ; \mathbb{R}^{2}\right)\right)$ with its dual space $H^{\prime}$, so that $V \hookrightarrow H \hookrightarrow V^{\prime}$ with dense and continuous embeddings. Moreover, we denote by $\|\cdot\|_{X}$ the norm in some space $X$, by $(\cdot, \cdot)$ the scalar product in $H$, and by $\langle\cdot, \cdot\rangle$ the duality pairing between $V$ and $V^{\prime}$. Hence, for any $\zeta \in V^{\prime}$, set

$$
\begin{gathered}
\zeta_{\Omega}:=\frac{1}{|\Omega|}\langle\zeta, 1\rangle, \\
\mathcal{V}^{\prime}:=\left\{\zeta \in V^{\prime}: \zeta_{\Omega}=0\right\}, \quad \mathcal{V}:=V \cap \mathcal{V}^{\prime} .
\end{gathered}
$$

The above notation $\mathcal{V}^{\prime}$ is just suggested for the sake of convenience; indeed, we mainly see $\mathcal{V}, \mathcal{V}^{\prime}$ as (closed) subspaces of $V, V^{\prime}$, inheriting their norms, rather than as a couple of spaces in duality.

From now on, for simplicity and without any loss of generality, we suppose that the coefficients in (2.19)-(2.21) are $k=m=c_{0}=\lambda=\mu=\ell / \vartheta_{c}=1$. Next, in order to give a precise formulation of our problem (2.19)-(2.21), we define here the realization 
of the Laplace operator with Neumann homogeneous boundary conditions, that is, the operator

$$
\mathcal{B}: V \rightarrow V^{\prime}, \quad\langle\mathcal{B} u, v\rangle=\int_{\Omega} \nabla u \cdot \nabla v, \quad u, v \in V .
$$

Clearly, $\mathcal{B}$ maps $V$ onto $\mathcal{V}^{\prime}$ and its restriction to $\mathcal{V}$ is an isomorphism of $\mathcal{V}$ onto $\mathcal{V}^{\prime}$.

Define $W:=\left\{w \in H^{2}(\Omega): \partial_{\mathbf{n}} w=0 \quad\right.$ on $\left.\Gamma\right\}$, where $\partial_{\mathbf{n}}$ is the derivative with respect to the outward normal derivative to $\Gamma$ and make the following assumptions on the data.

Hypothesis 3.1. We assume that $\varrho_{1}>\varrho_{2}>0, \nu \geq 0$, and suppose that

$\varphi: \mathbb{R}^{2} \rightarrow[0,+\infty]$ is proper, convex, lower semicontinuous and $\varphi(\mathbf{0})=0$,

$\mathcal{D}(\varphi)$ bounded if $\nu=0$,

$\gamma(r):=\exp (r)$ for $r \in \mathbb{R}$,

$\vartheta_{0} \in L^{1}(\Omega), \quad \vartheta_{0}>0 \quad$ a.e. in $\Omega, \quad w_{0}:=\gamma^{-1}\left(\vartheta_{0}\right) \in H$,

$\boldsymbol{\beta}_{0}=\left(\beta_{1}^{0}, \beta_{2}^{0}\right) \in \mathcal{D}(\varphi), \quad \nu \beta_{1}^{0}, \nu \beta_{2}^{0} \in V, \quad p_{0} \in V$,

$R \in L^{2}\left(Q_{T}\right) \cap L^{1}\left(0, T ; L^{\infty}(\Omega)\right), \quad \Pi \in L^{\infty}\left(\Sigma_{T}\right)$.

Then, we introduce the functions $\mathcal{R} \in L^{2}\left(0, T ; V^{\prime}\right)$ such that

$$
\langle\mathcal{R}(t), v\rangle=\int_{\Omega} R(t) v+\int_{\partial \Omega} \Pi(t) v_{\mid \partial \Omega}, \quad v \in V, \quad \text { for a.e. } t \in[0, T] .
$$

Moreover, we denote by $\partial \varphi$ the subdifferential in $\mathbb{R}^{2} \times \mathbb{R}^{2}$ of the convex analysis and note that $\partial \varphi$ is maximal monotone and that $\partial \varphi(\mathbf{0}) \ni \mathbf{0}$ (see, e.g., [2] and [10] for the general theory). The same symbol $\partial \varphi$ will be used for the maximal monotone operators induced on $L^{2}$-spaces.

REMARK 3.2. Here in our analysis we have chosen to treat the Neumann homogeneous boundary conditions on $p$; that is, we consider (2.24) with $\alpha_{p}=0$. It is not difficult, however, to treat the boundary conditions (2.24) with $\alpha_{p}>0$ with the same techniques used here. Regarding, instead, the absolute temperature $\vartheta$, we work here with Neumann nonhomogeneous boundary conditions on $\log \vartheta$, which means we consider (2.23) in case $\alpha_{\vartheta}>0$ and $\vartheta_{\text {ext }}=0$. Other types of boundary conditions such as Dirichlet nonhomogeneous boundary conditions on $\log \vartheta$ could also be taken into account without any difficulties in our analysis. Regarding instead the more sophisticated boundary conditions on $p$ detailed in Remark 2.3. a more careful analysis should be performed in order to include them in our results, but we do not want to face this problem in this paper.

Then, we are ready to introduce the variational formulation of our problem as follows.

Problem $(\mathrm{P})$. Find $\left(w, \beta_{1}, \beta_{2}\right)$ and $\left(\vartheta, \xi_{1}, \xi_{2}, p\right)$ with the regularities

$$
\begin{aligned}
& w \in H^{1}\left(0, T ; V^{\prime}\right) \cap L^{2}(0, T ; V), \quad \vartheta \in L^{5 / 3}\left(Q_{T}\right), \\
& \beta_{1}, \beta_{2} \in H^{1}(0, T ; H), \quad \nu \beta_{1}, \nu \beta_{2} \in L^{\infty}(0, T ; V) \cap L^{5 / 3}\left(0, T ; W^{2,5 / 3}(\Omega)\right), \\
& \boldsymbol{\beta}=\left(\beta_{1}, \beta_{2}\right) \in \mathcal{D}(\varphi) \quad \text { a.e. in } Q_{T}, \\
& \xi_{1}, \xi_{2} \in L^{5 / 3}\left(Q_{T}\right), \quad p \in C^{0}([0, T] ; V) \cap L^{2}(0, T ; W) \cap H^{1}(0, T ; H),
\end{aligned}
$$


satisfying

$$
\begin{aligned}
& \partial_{t} p+\varrho_{1} \partial_{t} \beta_{1}+\varrho_{2} \partial_{t} \beta_{2}+\mathcal{B} p=0 \quad \text { a.e. in } Q_{T}, \\
& \partial_{t} w+\partial_{t} \beta_{1}+\mathcal{B} w=\mathcal{R} \quad \text { in } V^{\prime} \text { a.e. in }[0, T], \\
& \partial_{t} \boldsymbol{\beta}+\nu\left(\begin{array}{c}
\mathcal{B} \beta_{1} \\
\mathcal{B} \beta_{2}
\end{array}\right)+\boldsymbol{\xi}-p\left(\begin{array}{c}
\varrho_{1} \\
\varrho_{2}
\end{array}\right)=\left(\begin{array}{c}
\vartheta-\vartheta_{c} \\
0
\end{array}\right) \quad \text { a.e. in } Q_{T}, \\
& \vartheta=\gamma(w), \quad \boldsymbol{\xi}=\left(\xi_{1}, \xi_{2}\right) \in \partial \varphi(\boldsymbol{\beta}) \quad \text { a.e. in } Q_{T},
\end{aligned}
$$

and such that

$$
\begin{aligned}
& w(0)=w_{0}, \quad p(0)=p_{0} \quad \text { a.e. in } \Omega, \\
& \boldsymbol{\beta}(0)=\left(\beta_{1}(0), \beta_{2}(0)\right)=\boldsymbol{\beta}_{0} \quad \text { a.e. in } \Omega .
\end{aligned}
$$

REMARK 3.3. Notice that testing formally equation (3.15) by 1 one can immediately deduce that the "total mass" of $p+\boldsymbol{\beta}^{\varrho}$, where $\boldsymbol{\beta}^{\varrho}:=\left(\varrho_{1} \beta_{1}, \varrho_{2} \beta_{2}\right)$, is conserved during the time interval $[0, T]$. Indeed, we have

$$
\int_{\Omega}\left(p+\varrho_{1} \beta_{1}+\varrho_{2} \beta_{2}\right)(t)=\int_{\Omega}\left(p+\varrho_{1} \beta_{1}+\varrho_{2} \beta_{2}\right)(0)
$$

for all $t \in[0, T]$. The physical meaning of this relation is that the mass of water either liquid or solid contained in the domain $\Omega$ is constant, due to the water-tight boundary condition we have chosen (cf. (2.24) with $\alpha_{p}=0$ ). Finally, let us note that, by interpolation, from (3.12) it follows that $w \in C^{0}([0, T] ; H)$ and so all three boundary conditions in (3.19) $-(3.20)$ hold in $H$.

REMARK 3.4. Obviously, an admissible convex function satisfying assumption (3.5) is, e.g., $\varphi=I_{K}, K$ being the convex set of $\mathbb{R}^{2}$ defined in the Introduction.

REMARK 3.5. Let us underline here (cf. also [6, Remark 3.1]) some regularity properties of possible solutions to Problem (P). First of all, let us recall the GagliardoNirenberg inequality in $3 \mathrm{D}$, that is,

$$
\|v\|_{L^{p}(\Omega)} \leq c\|v\|_{V}^{\alpha}\|v\|_{H}^{1-\alpha} \quad \forall v \in V,
$$

for $\frac{1}{p}=\frac{\alpha}{6}+\frac{1-\alpha}{2}$ and for some positive constant $c$. Hence, if we are able to prove that $\vartheta^{1 / 2} \in L^{\infty}(0, T ; H) \cap L^{2}(0, T ; V)$, then it follows that

$$
\vartheta \in L^{5 / 3}(Q) \text {. }
$$

REMARK 3.6. Note that in our analysis we could also consider a more general form of our equation (3.17); we can treat the case in which an antimonotone, but smooth, contribution is added to the monotone one given by $\partial \varphi$. Indeed, we can cover with our analysis the case in which (3.17) is substituted by the more general inclusion:

$$
\partial_{t} \boldsymbol{\beta}+\nu\left(\begin{array}{c}
\mathcal{B} \beta_{1} \\
\mathcal{B} \beta_{2}
\end{array}\right)+\partial \varphi(\boldsymbol{\beta})+\sigma^{\prime}(\boldsymbol{\beta})-p\left(\begin{array}{l}
\varrho_{1} \\
\varrho_{2}
\end{array}\right) \ni\left(\begin{array}{c}
\vartheta-\vartheta_{c} \\
0
\end{array}\right) \quad \text { a.e. in } Q_{T},
$$

where $\sigma \in C^{1,1}\left(\mathbb{R}^{2}\right)$ is a, possibly antimonotone, contribution coming from the possibly nonconvex part $\sigma(\boldsymbol{\beta})$ in the free energy functional (2.5). The example we have in mind is the one given by the "classical" double-well potential.

The aim of the following Section 5.1 is to prove one of our main results, that is, the following global existence theorem. 
Theorem 3.7. Let Hypothesis 3.1 hold true and let $T$ be a positive final time. Then Problem $(\mathrm{P})$ has a solution at least on the whole time interval $[0, T]$.

Remark 3.8. The problem of finding also a uniqueness result for Problem (P) is still open and is strictly related to the possibility of finding more regularity on the $\vartheta$ component of the solution to Problem (P). The reader can refer also to [6] and 7 for further comments on this topic in case the voids are not admissible and only the two equations (3.16) and (3.17) (with $p=0, \varphi=I_{C}$, and $C=\left\{\left(\beta_{1}, \beta_{2}\right) \in[0,1] \mid \beta_{1}+\beta_{2}=1\right\}$ ) are coupled.

The proof of Theorem 3.7 is based on the following scheme, which follows the idea of [6]: we first solve an approximating problem in which $\gamma$ is substituted by a Lipschitzcontinuous function, and then we make a priori estimates (independent of the approximation parameter), which will allow us to pass to the limit by means of compactness and monotonicity arguments. In view of these considerations, let us state here a preliminary result (whose proof will be given in Section 4).

Consider the following assumptions on the data.

Hypothesis 3.9. Let (3.5)-(3.6) and (3.9) hold true and assume moreover that:

$$
\begin{aligned}
& \delta:=\gamma^{-1}: \mathbb{R} \rightarrow \mathbb{R}^{2} \text { is a maximal monotone operator } \\
& \text { with Lipschitz continuous inverse graph } \gamma, \\
& w_{0} \in H, \quad \mathcal{R} \in L^{2}\left(0, T ; V^{\prime}\right),
\end{aligned}
$$

where $\mathcal{R}$ is defined in (3.11).

Theorem 3.10. Suppose now that Hypothesis 3.9 holds true. Then Problem (P) has at least a solution $\left(w, \beta_{1}, \beta_{2}\right),\left(\vartheta, \xi_{1}, \xi_{2}, p\right)$ satisfying the following regularity properties:

$$
\begin{aligned}
& w, \vartheta \in C^{0}([0, T] ; H) \cap L^{2}(0, T ; V), \\
& \nu \beta_{1}, \nu \beta_{2} \in L^{2}(0, T ; W), \quad \xi_{1}, \xi_{2} \in L^{2}(0, T ; H) .
\end{aligned}
$$

Moreover, the components $w, \beta_{1}, \beta_{2}, p$, and $\vartheta$ of such a solution are uniquely determined.

REMARK 3.11. Observe that the main advantage of taking the entropy balance equation (3.16) instead of the internal energy balance equation is that once one has solved the problem in some sense and has found the temperature $\vartheta:=\gamma(w)$, it is automatically positive because it stands in the image of the function $\gamma$ (cf. (3.7)). Indeed in many cases it is difficult to deduce this fact only from the internal energy balance equation (cf., e.g., [13] in order to see one example of these difficulties). Let us note that within the small perturbations assumption the entropy balance and the classical heat equation are equivalent in mechanical terms (cf. [6, 7]).

Moreover, in the same framework as above, let us recall the following inequality, holding in $\Omega$ introduced above (cf. (3.2)):

$$
\left\|v-v_{\Omega}\right\|_{H} \leq c\|\nabla v\|_{H} \quad \forall v \in V,
$$

for some constants $c>0$, depending only on $\Omega$. Inequality (3.28) is one form of the standard Poincaré-Wirtinger inequality. Finally, we will make use of the following elementary 
inequality:

$$
a b \leq \eta a^{2}+\frac{1}{4 \eta} b^{2}, \quad \forall a, b \in \mathbb{R} \quad \forall \eta>0 .
$$

4. Proof of Theorem 3.10. In this section we assume Hypothesis 3.9 and we prove Theorem 3.10. Here we are going to prove the existence and uniqueness of solutions to Problem $(\mathrm{P})^{\varepsilon}$ in the time interval $[0, T]$, using a fixed point technique of contractive type.

In order to develop the proof in a rigorous way, we introduce here the Lipschitz continuous Yosida-Moreau approximation $\partial \varphi^{\varepsilon}=\left(\varphi^{\varepsilon}\right)^{\prime}$ (cf. [10, Prop. 2.11, p. 39]) of $\partial \varphi$, we first solve the Problem $(\mathrm{P})$ with $\partial \varphi^{\varepsilon}$ instead of $\partial \varphi$ (call it $(\mathrm{P})^{\varepsilon}$ in this section) and then we perform a priori estimates independent of $\varepsilon$ and pass to the limit as $\varepsilon \searrow 0$ recovering a solution to Problem $(\mathrm{P})$. Moreover, we denote by $c$ the positive constants (may be different from line to line), depending on the data of the problem, but not on $\varepsilon$. Then we are ready to introduce the variational formulation of our approximating $\operatorname{Problem}(\mathrm{P})^{\varepsilon}$ as follows.

Problem $(\mathrm{P})^{\varepsilon}$. Find $\left(w^{\varepsilon}, \beta_{1}^{\varepsilon}, \beta_{2}^{\varepsilon}\right)$ and $\left(\vartheta^{\varepsilon}, \xi_{1}^{\varepsilon}, \xi_{2}^{\varepsilon}, p^{\varepsilon}\right)$ with the regularities

$$
\begin{aligned}
& w^{\varepsilon}, \vartheta^{\varepsilon} \in H^{1}\left(0, T ; V^{\prime}\right) \cap L^{2}(0, T ; V), \\
& \boldsymbol{\beta}^{\varepsilon}=\left(\beta_{1}^{\varepsilon}, \beta_{2}^{\varepsilon}\right) \in\left(H^{1}(0, T ; H)\right)^{2}, \quad \nu \beta_{1}^{\varepsilon}, \nu \beta_{2}^{\varepsilon} \in L^{\infty}(0, T ; V) \cap L^{2}(0, T ; W), \\
& \xi_{1}^{\varepsilon}, \xi_{2}^{\varepsilon} \in L^{2}\left(Q_{T}\right), \quad p^{\varepsilon} \in C^{0}([0, T] ; V) \cap L^{2}(0, T ; W) \cap H^{1}(0, T ; H),
\end{aligned}
$$

satisfying

$$
\begin{aligned}
& \partial_{t} p^{\varepsilon}+\varrho_{1} \partial_{t} \beta_{1}^{\varepsilon}+\varrho_{2} \partial_{t} \beta_{2}^{\varepsilon}+\mathcal{B} p^{\varepsilon}=0 \quad \text { a.e. in } Q_{T}, \\
& \partial_{t} w^{\varepsilon}+\partial_{t} \beta_{1}^{\varepsilon}+\mathcal{B} w^{\varepsilon}=\mathcal{R} \quad \text { in } V^{\prime}, \text { a.e. in }[0, T], \\
& \partial_{t} \boldsymbol{\beta}^{\varepsilon}+\nu\left(\begin{array}{c}
\mathcal{B} \beta_{1}^{\varepsilon} \\
\mathcal{B} \beta_{2}^{\varepsilon}
\end{array}\right)+\boldsymbol{\xi}^{\varepsilon}-p^{\varepsilon}\left(\begin{array}{c}
\varrho_{1} \\
\varrho_{2}
\end{array}\right)=\left(\begin{array}{c}
\vartheta^{\varepsilon}-\vartheta_{c} \\
0
\end{array}\right) \quad \text { a.e. in } Q_{T}, \\
& \vartheta^{\varepsilon}=\gamma\left(w^{\varepsilon}\right), \quad \boldsymbol{\xi}^{\varepsilon}=\left(\xi_{1}^{\varepsilon}, \xi_{2}^{\varepsilon}\right)=\left(\varphi^{\varepsilon}\right)^{\prime}\left(\boldsymbol{\beta}^{\varepsilon}\right) \quad \text { a.e. in } Q_{T},
\end{aligned}
$$

and such that

$$
\begin{aligned}
& w^{\varepsilon}(0)=w_{0}, \quad p^{\varepsilon}(0)=p_{0} \quad \text { a.e. in } \Omega, \\
& \boldsymbol{\beta}^{\varepsilon}(0)=\left(\beta_{1}^{\varepsilon}(0), \beta_{2}^{\varepsilon}(0)\right)=\boldsymbol{\beta}_{0} \quad \text { a.e. in } \Omega .
\end{aligned}
$$

Now we start here by proving the existence of solutions to $(\mathrm{P})^{\varepsilon}$, by means of a contraction argument.

Existence of solutions to $(\mathrm{P})^{\varepsilon}, \varepsilon \in(0, \bar{\varepsilon}]$. Let us take $\bar{t} \in[0, T]$ (we will choose it later) and define

$$
\mathcal{X}:=\left\{(r, s) \in\left(H^{1}(0, \bar{t} ; H)\right)^{2}:(r, s) \in \mathcal{D}(\varphi)\right\} .
$$

First fix $\left(\bar{\beta}_{1}, \bar{\beta}_{2}\right) \in \mathcal{X}$ in the equations (4.4) and (4.5); then, by well-known results (cf. [1. Lemma 6.3] and also (3.10) $)$, we also find a unique solution $w:=\mathcal{T}_{1}\left(\bar{\beta}_{1}, \overline{\beta_{2}}\right) \in$ $H^{1}(0, T ; H) \cap C^{0}([0, T] ; V) \cap L^{2}\left(0, T ; H^{2}(\Omega)\right)$ of (4.5) and a solution $p:=\mathcal{T}_{2}\left(\bar{\beta}_{1}, \bar{\beta}_{2}\right) \in$ $H^{1}(0, T ; H) \cap C^{0}([0, T] ; V) \cap L^{2}(0, T ; W)$ to (4.4). Then, if we take these values of $w$ and $p$ in (4.6) in place of $w^{\varepsilon}, p^{\varepsilon}$, again by standard results, we can find the solution $\boldsymbol{\beta}=\left(\beta_{1}, \beta_{2}\right)$ to (4.6). 
In this way, we have defined an operator $\mathcal{T}: \mathcal{X} \rightarrow \mathcal{X}$ such that $\left(\beta_{1}, \beta_{2}\right)=: \mathcal{T}\left(\bar{\beta}_{1}, \bar{\beta}_{2}\right)$. What we have to do now is to prove that $\mathcal{T}$ is a contraction mapping on $\mathcal{X}$ for a sufficiently small $\bar{t} \in[0, T]$. In order to prove that $\mathcal{T}$ is contractive, let us proceed by steps and forget about the apices $\varepsilon$.

First step. Let $\left({\overline{\beta_{1}}}^{i},{\overline{\beta_{2}}}^{i}\right) \in \mathcal{X}(i=1,2), p^{i}=\mathcal{T}_{2}\left({\overline{\beta_{1}}}^{i},{\overline{\beta_{2}}}^{i}\right), w^{i}=\mathcal{T}_{1}\left({\overline{\beta_{1}}}^{i},{\overline{\beta_{2}}}^{i}\right)$, and $\left(\beta_{1}^{i}, \beta_{2}^{i}\right)=\mathcal{T}\left(\bar{\beta}_{1}{ }^{i}, \bar{\beta}_{2}{ }^{i}\right)$. Then, writing two times (4.5) with $\left(\bar{\beta}_{1}{ }^{i}, \bar{\beta}_{2}{ }^{i}\right)(i=1,2)$, making the difference, testing the resulting equation with $\left(w^{1}-w^{2}\right)$, and integrating on $(0, t)$ with $t \in[0, \bar{t}]$, we get the following inequality:

$$
\begin{aligned}
& \left\|w^{1}(t)-w^{2}(t)\right\|_{H}^{2}+\left\|w^{1}-w^{2}\right\|_{L^{2}(0, t ; V)}^{2} \\
& \leq C_{1}\left(\left\|\left(\bar{\beta}_{1}{ }^{1}\right)_{t}-\left(\bar{\beta}_{1}{ }^{2}\right)_{t}\right\|_{L^{2}(0, t ; H)}^{2}+\left\|\left(\bar{\beta}_{2}{ }^{1}\right)_{t}-\left({\overline{\beta_{2}}}^{2}\right)_{t}\right\|_{L^{2}(0, t ; H)}^{2}\right),
\end{aligned}
$$

for some positive constant $C_{1}$ independent of $t$.

Second step. We now write (4.4) with $\left(\bar{\beta}_{1}{ }^{i}, \bar{\beta}_{2}{ }^{i}\right)$, make the difference, test the resulting equation with $p^{1}-p^{2}$, and integrate on $(0, t)$ with $t \in[0, \bar{t}]$. Then we get the following inequality:

$$
\begin{aligned}
& \left\|\left(p^{1}-p^{2}\right)(t)\right\|_{H}^{2}+\left\|p^{1}-p^{2}\right\|_{L^{2}(0, t ; V)}^{2} \\
& \leq C_{2}\left(\left\|\left({\overline{\beta_{1}}}^{1}\right)_{t}-\left({\overline{\beta_{1}}}^{2}\right)_{t}\right\|_{L^{2}(0, t ; H)}^{2}+\left\|\left({\overline{\beta_{2}}}^{1}\right)_{t}-\left({\overline{\beta_{2}}}^{2}\right)_{t}\right\|_{L^{2}(0, t ; H)}^{2}\right),
\end{aligned}
$$

for some positive constant $C_{2}$ independent of $t$.

Third step. Let us take $\vartheta^{i}=\gamma\left(w^{i}\right)(i=1,2)$ and write equation (4.6) for $\vartheta^{i}$ and $u^{i}$, make the difference between the two equations written for $i=1$ and $i=2$, and test the resulting vectorial equation by the vector $\left(\left(\beta_{1}^{1}\right)_{t}-\left(\beta_{1}^{2}\right)_{t},\left(\beta_{2}^{1}\right)_{t}-\left(\beta_{2}^{2}\right)_{t}\right)$. Summing up the two lines and integrating on $(0, t)$ with $t \in[0, \bar{t}]$, we have

$$
\begin{aligned}
& \sum_{j=1}^{2}\left\|\left(\beta_{j}^{1}\right)_{t}-\left(\beta_{j}^{2}\right)_{t}\right\|_{L^{2}(0, t ; H)}^{2}+\nu \sum_{j=1}^{2}\left\|\nabla\left(\beta_{j}^{1}-\beta_{j}^{2}\right)(t)\right\|_{H}^{2} \\
& \leq \int_{Q_{T}}\left|\left(p^{1}-p^{2}\right)\left(\varrho_{1}\left(\left(\beta_{1}^{1}\right)_{t}-\left(\beta_{1}^{2}\right)_{t}\right)+\varrho_{2}\left(\left(\beta_{2}^{1}\right)_{t}-\left(\beta_{2}^{2}\right)_{t}\right)\right)\right| \\
& \quad+\frac{1}{4} \sum_{j=1}^{2}\left\|\left(\beta_{j}^{1}\right)_{t}-\left(\beta_{j}^{2}\right)_{t}\right\|_{L^{2}(0, t ; H)}^{2}+2\left\|\vartheta^{1}-\vartheta^{2}\right\|_{L^{2}(0, t ; H)}^{2} \\
& \leq \frac{1}{2} \sum_{j=1}^{2}\left\|\left(\beta_{j}^{1}\right)_{t}-\left(\beta_{j}^{2}\right)_{t}\right\|_{L^{2}(0, t ; H)}^{2}+2\left\|\vartheta^{1}-\vartheta^{2}\right\|_{L^{2}(0, t ; H)}^{2}+2\left\|p^{1}-p^{2}\right\|_{L^{2}(0, t ; H)}^{2} .
\end{aligned}
$$

Moreover, using the assumption (3.24) on $\gamma$, we get the following inequality:

$$
\begin{aligned}
& \frac{1}{2} \sum_{j=1}^{2}\left\|\left(\beta_{j}^{1}\right)_{t}-\left(\beta_{j}^{2}\right)_{t}\right\|_{L^{2}(0, t ; H)}^{2}+\nu \sum_{j=1}^{2}\left\|\nabla\left(\beta_{j}^{1}-\beta_{j}^{2}\right)(t)\right\|_{H}^{2} \\
& \leq 2 t\left(\left\|w^{1}-w^{2}\right\|_{C^{0}([0, t] ; H)}^{2}+\left\|p^{1}-p^{2}\right\|_{C^{0}([0, t] ; H)}^{2}\right) .
\end{aligned}
$$


Fourth step. Summing up the two inequalities (4.11) and (4.12), and using (4.10), we get

$$
\begin{aligned}
& \sum_{j=1}^{2}\left\|\left(\beta_{j}^{1}\right)_{t}-\left(\beta_{j}^{2}\right)_{t}\right\|_{L^{2}(0, t ; H)}^{2}+\nu \sum_{j=1}^{2}\left\|\nabla\left(\beta_{j}^{1}-\beta_{j}^{2}\right)(t)\right\|_{H}^{2} \\
& \leq C_{3} t \sum_{j=1}^{2}\left\|\left(\beta_{j}^{1}\right)_{t}-\left(\beta_{j}^{2}\right)_{t}\right\|_{L^{2}(0, t ; H)}^{2},
\end{aligned}
$$

for some positive constant $C_{3}$ independent of $t$. Hence, choosing $t$ sufficiently small (this is our $\bar{t}$, we recover the contractive property of $\mathcal{T}$. Moreover, applying the Banach fixed point theorem to $\mathcal{T}$, we get a unique solution for Problem $(\mathrm{P})^{\varepsilon}$ on the time interval $[0, \bar{t}]$. Then, it is not difficult to check that

$$
\left|\mathcal{T}\left({\overline{\beta_{1}}}_{1}{ }^{1},{\overline{\beta_{2}}}^{1}\right)^{k}-\mathcal{T}\left({\overline{\beta_{1}}}^{2},{\overline{\beta_{2}}}^{2}\right)^{k}\right|_{\left(H^{1}(0, T ; H)\right)^{2}} \leq \frac{\left(C_{3} T\right)^{k}}{k !}\left|\left(\bar{\beta}_{1}{ }^{1},{\overline{\beta_{2}}}^{1}\right)-\left({\overline{\beta_{1}}}^{2},{\overline{\beta_{2}}}^{2}\right)\right|_{\left(H^{1}(0, T ; H)\right)^{2}}
$$

for any power $\mathcal{T}^{k}$ of $\mathcal{T}$. Hence, one can find $k$ such that $\left(C_{3} T\right)^{k}<k$ ! and consequently the corresponding $\mathcal{T}^{k}$ is a contraction and admits a unique fixed point, which is the searched solution to Problem $(\mathrm{P})^{\varepsilon}$ on $[0, T]$.

Estimates and passage to the limit. We now perform uniform in $\varepsilon$ estimates on the solution to Problem $(\mathrm{P})^{\varepsilon}$, which allow us to pass to the limit as $\varepsilon \searrow 0$.

First estimate. Test (4.4) by $p$, (4.5) by $\vartheta=\gamma(w)$, and (4.6) by $\partial_{t} \boldsymbol{\beta}$. Then, sum up the result of the first two tests with the sum of the two components of the vectorial equation found by the third test. This, after an integration in time on $(0, t)$, with $t \in[0, T]$, thanks to a cancellation of two integrals, leads to

$$
\begin{aligned}
& \frac{1}{2}\|p(t)\|_{H}^{2}+\|\nabla p\|_{L^{2}(0, t ; H)}^{2}+\int_{\Omega} \widehat{\gamma}(w(t))+c_{\gamma} \int_{0}^{t} \int_{\Omega}|\nabla \vartheta|^{2}+\sum_{j=1}^{2}\left\|\partial_{t} \beta_{j}\right\|_{L^{2}(0, t ; H)}^{2} \\
& +\frac{\nu}{2} \sum_{j=1}^{2}\left\|\nabla \beta_{j}(t)\right\|_{H}^{2}+\int_{\Omega} \varphi^{\varepsilon}(\boldsymbol{\beta}(t)) \leq \frac{1}{2}\left\|p_{0}\right\|_{H}^{2}+\int_{\Omega} \widehat{\gamma}\left(w_{0}\right)+\int_{0}^{t}\langle\mathcal{R}, \vartheta\rangle \\
& +\frac{\nu}{2} \sum_{j=1}^{2}\left\|\nabla \beta_{j}(0)\right\|_{H}^{2}+\int_{\Omega} \varphi^{\varepsilon}\left(\boldsymbol{\beta}_{0}\right)+\int_{0}^{t} \int_{\Omega} \vartheta_{c} \partial_{t} \beta_{1}
\end{aligned}
$$

Now, using (3.9), (3.24)-(3.25), and the Schwarz inequality with (3.29), we get:

$$
\begin{aligned}
& \|p(t)\|_{H}^{2}+\|\nabla p\|_{L^{2}(0, t ; H)}^{2}+\int_{\Omega} \vartheta(t)+\int_{0}^{t} \int_{\Omega}|\nabla \vartheta(s)|^{2} d s+\sum_{j=1}^{2}\left\|\partial_{t} \beta_{j}\right\|_{L^{2}(0, t ; H)}^{2} \\
& +\nu \sum_{j=1}^{2}\left\|\nabla \beta_{j}(t)\right\|_{H}^{2}+\int_{\Omega} \varphi^{\varepsilon}(\boldsymbol{\beta}(t)) \leq c+c_{\eta}\|R\|_{L^{2}\left(0, t ; V^{\prime}\right)}^{2}+\eta\left\|\vartheta^{\varepsilon}\right\|_{L^{2}(0, t ; V)}^{2}
\end{aligned}
$$


holding true for all $\eta>0$ and for some positive $c_{\eta}$. Finally, using (3.28) and (3.25), and choosing $\eta$ sufficiently small, we get:

$$
\begin{aligned}
& \|p(t)\|_{H}^{2}+\|\nabla p\|_{L^{2}(0, t ; H)}^{2}+\|\vartheta\|_{L^{2}(0, t ; V)}^{2}+\sum_{j=1}^{2}\left\|\partial_{t} \beta_{j}\right\|_{L^{2}(0, t ; H)}^{2} \\
& +\nu \sum_{j=1}^{2}\left\|\nabla \beta_{j}(t)\right\|_{H}^{2}+\int_{\Omega} \varphi^{\varepsilon}(\boldsymbol{\beta}(t)) \leq c .
\end{aligned}
$$

Then, testing (4.5) with $w$ and using (4.15), it is a standard matter to deduce the following bound:

$$
\|w\|_{L^{\infty}(0, T ; H) \cap L^{2}(0, T ; V) \cap H^{1}\left(0, T ; V^{\prime}\right)} \leq c,
$$

from which, using assumptions (3.24) and (3.9), we also deduce

$$
\|\vartheta\|_{L^{\infty}(0, T ; H) \cap L^{2}(0, T ; V) \cap H^{1}\left(0, T ; V^{\prime}\right)}+\|p\|_{H^{1}(0, T ; H) \cap L^{\infty}(0, T ; V) \cap L^{2}(0, T ; W)} \leq c .
$$

Second estimate. Now test (4.6) by $\boldsymbol{\xi}^{\varepsilon}$. Note that the term

$$
\left(\nabla \boldsymbol{\beta}^{\varepsilon}(t), \nabla \boldsymbol{\xi}^{\varepsilon}(t)\right)=\left(\nabla \boldsymbol{\beta}^{\varepsilon}(t), \nabla\left(\varphi^{\varepsilon}\right)^{\prime}\left(\boldsymbol{\beta}^{\varepsilon}(t)\right)\right)
$$

is nonnegative due to the monotonicity of $\left(\varphi^{\varepsilon}\right)^{\prime}$. Hence, due to estimate (4.15) and (4.17), we obtain

$$
\left\|\xi_{i}^{\varepsilon}\right\|_{L^{2}(0, T ; H)} \leq c, \quad i=1,2
$$

and also

$$
\left\|\nu \beta_{i}\right\|_{L^{2}(0, T ; W)} \leq c, \quad i=1,2,
$$

by comparison in (3.17) and by standard regularity results for elliptic equations.

Passage to the limit. As we have just mentioned, we want to conclude the proof of Theorem 3.10 by passing to the limit in Problem $(\mathrm{P})^{\varepsilon}$ as $\varepsilon \searrow 0$ using the previous uniform (in $\varepsilon$ ) estimates on its solution and exploiting some compactness-monotonicity arguments. First let us list the weak or weak-star convergence coming directly from the previous estimates and well-known weak-compactness results. Note that the following convergences hold only up to a subsequence of $\varepsilon$ tending to 0 (let us say $\varepsilon_{k} \searrow 0$ ). We denote it again with $\varepsilon$ only for simplicity of notation. From the estimates (4.15)-(4.17), (4.18)-(4.19), we deduce that there exist $\left(w, \bar{\vartheta}, \beta_{j}, p, \xi_{j}\right), j=1,2$, such that

$$
\begin{aligned}
w^{\varepsilon} \rightarrow w & \text { weakly star in } H^{1}\left(0, T ; V^{\prime}\right) \cap L^{2}(0, T ; V), \\
\vartheta^{\varepsilon} \rightarrow \bar{\vartheta} & \text { weakly star in } H^{1}\left(0, T ; V^{\prime}\right) \cap L^{2}(0, T ; V), \\
\beta_{j}^{\varepsilon} \rightarrow \beta_{j} & \text { weakly in } H^{1}(0, T ; H)(j=1,2), \\
\nu \beta_{j}^{\varepsilon} \rightarrow \nu \beta_{j} & \text { weakly star in } L^{\infty}(0, T ; V) \cap L^{2}(0, T ; W)(j=1,2), \\
p^{\varepsilon} \rightarrow p & \text { weakly star in } H^{1}(0, T ; H) \cap L^{\infty}(0, T ; V) \cap L^{2}(0, T ; W), \\
\xi_{j}^{\varepsilon} \rightarrow \xi_{j} & \text { weakly in } L^{2}\left(Q_{T}\right) \quad(j=1,2) .
\end{aligned}
$$


Moreover, employing the Aubin-Lions lemma (cf. [18, p. 58]) and [21, Cor. 8, p. 89], we also get

$$
\begin{aligned}
w^{\varepsilon} \rightarrow w & \text { strongly in } L^{2}(0, T ; H), \quad \text { and hence a.e. in } Q_{T}, \\
\vartheta^{\varepsilon} \rightarrow \bar{\vartheta} & \text { strongly in } L^{2}(0, T ; H), \quad \text { and hence a.e. in } Q_{T}, \\
\beta_{j}^{\varepsilon} \rightarrow \beta_{j} & \text { strongly in } C^{0}([0, T] ; H) \cap L^{2}(0, T ; V) \quad \text { if } \nu>0 \quad(j=1,2), \\
p^{\varepsilon} \rightarrow p & \text { strongly in } C^{0}([0, T] ; H) \cap L^{2}(0, T ; V) \quad \text { and hence a.e. in } Q_{T} .
\end{aligned}
$$

Note that convergences (4.21), (4.26), and relation (5.10) imply immediately (cf. [18, Lemme 1.3, p. 12]) the convergence

$$
\vartheta^{\varepsilon} \rightarrow \bar{\vartheta}=\gamma(w) \quad \text { a.e. in } Q_{T} .
$$

Finally, it remains to prove the identification of the maximal monotone graph $\partial \varphi$, i.e.

$$
\boldsymbol{\xi} \in \partial \varphi(\boldsymbol{\beta}) \text { a.e. in } Q_{T},
$$

with $\boldsymbol{\xi}=\left(\xi_{1}, \xi_{2}\right)$ and $\xi_{j}(j=1,2)$, which are the weak limits defined in (4.25). In order to do that we should verify that

$$
\limsup _{\varepsilon \searrow 0} \int_{0}^{T}\left(\boldsymbol{\xi}^{\varepsilon}, \boldsymbol{\beta}^{\varepsilon}\right) \leq \int_{0}^{T}(\boldsymbol{\xi}, \boldsymbol{\beta}) .
$$

The proof here is split into two parts.

Case $\nu>0$. From (4.28) and (4.25) we immediately deduce that (5.32) is verified.

Case $\nu=0$. In this case we can prove that $\boldsymbol{\beta}^{\varepsilon}$ is a Cauchy sequence in $C^{0}([0, T] ; H)$. Indeed one can take the differences of equations (3.17) written for two different indices $\varepsilon$ and $\varepsilon^{\prime}$ and test it by the difference vector $\left(\boldsymbol{\beta}^{\varepsilon}-\boldsymbol{\beta}^{\varepsilon^{\prime}}\right)$. Take the differences of the two equations (3.15) integrated in time and test it by $p^{\varepsilon}-p^{\varepsilon^{\prime}}$. Summing up the two resulting equations and integrating over $(0, t), t \in(0, T)$, we get:

$$
\begin{aligned}
& \left\|p^{\varepsilon}-p^{\varepsilon^{\prime}}\right\|_{L^{2}(0, t ; H)}^{2}+\frac{1}{2}\left\|\left(\boldsymbol{\beta}^{\varepsilon}-\boldsymbol{\beta}^{\varepsilon^{\prime}}\right)(t)\right\|_{H}^{2} \leq \int_{Q_{t}}\left(\vartheta^{\varepsilon}-\vartheta^{\varepsilon^{\prime}}\right)\left(\beta_{1}^{\varepsilon}-\beta_{1}^{\varepsilon^{\prime}}\right) \\
& \leq \int_{0}^{t}\left\|\left(\vartheta^{\varepsilon}-\vartheta^{\varepsilon^{\prime}}\right)(s)\right\|_{H}\left\|\left(\beta_{1}^{\varepsilon}-\beta_{1}^{\varepsilon^{\prime}}\right)(s)\right\|_{H} d s .
\end{aligned}
$$

Applying now the Gronwall lemma [10, Lemme A.3], we get

$$
\left\|\left(\boldsymbol{\beta}^{\varepsilon}-\boldsymbol{\beta}^{\varepsilon^{\prime}}\right)(t)\right\|_{H}^{2} \leq c\left\|\vartheta^{\varepsilon}-\vartheta^{\varepsilon^{\prime}}\right\|_{L^{1}(0, T ; H)} \rightarrow 0 \quad \text { as } \varepsilon, \varepsilon^{\prime} \searrow 0 .
$$

Hence, we have that $\boldsymbol{\beta}^{\varepsilon} \rightarrow \boldsymbol{\beta}$ strongly in $C^{0}([0, T] ; H)$, which is sufficient in order to prove that (5.32) is satisfied.

Hence, in both cases, all these convergences with the identifications made above make us able to pass to the limit (as $\varepsilon \searrow 0$ or at least for a subsequence of it) in Problem $(\mathrm{P})^{\varepsilon}$ finding a solution to Problem $(\mathrm{P})$. Note that once we prove uniqueness, the convergences above will turn out to hold for every sequence $\varepsilon \searrow 0$ and not only up to a subsequence. 
Uniqueness. Assume that $\left(w^{1}, \boldsymbol{\beta}^{1}\right),\left(\vartheta^{1}, \boldsymbol{\xi}^{1}, p^{1}\right)$ and $\left(w^{2}, \boldsymbol{\beta}^{2}\right),\left(\vartheta^{2}, \boldsymbol{\xi}^{2}, p^{2}\right)$ are two solutions of Problem (P) corresponding to the same data, and let us denote $\bar{w}:=$ $w^{1}-w^{2}, \bar{p}:=p^{1}-p^{2}, \bar{\beta}_{j}:=\beta_{j}^{1}-\beta_{j}^{2}(j=1,2)$. Then form the differences from (3.15) written down for the first solution and (3.15), integrated in time and written down for the second solution. Then test the resulting equation by $\bar{p}$, test the difference between the equations (3.17) written in terms of $\boldsymbol{\beta}^{1}$ and $\boldsymbol{\beta}^{2}$ by $\left(\bar{\beta}_{1}, \bar{\beta}_{2}\right)$. Finally, integrate in time (3.16) (let us call it $1 *(3.16)$ ) and form the differences from $1 *(3.16)$ written down for the first solution and $1 *(3.16)$ written down for the second solution; then test the resulting equation by $\bar{w}$. Then sum up the three resulting equations and integrate it over $(0, t)$, using the maximal monotonicity of $\partial \varphi$ to get

$$
\begin{aligned}
& \|\bar{p}\|_{L^{2}(0, t ; H)}^{2}+\left\|\int_{0}^{t} \nabla \bar{p}\right\|_{H}^{2}+\sum_{j=1}^{2}\left\|\bar{\beta}_{j}(t)\right\|_{H}^{2}+\nu \sum_{j=1}^{2}\left\|\nabla \bar{\beta}_{j}\right\|_{L^{2}(0, t ; H)}^{2} \\
& +\|\bar{w}\|_{L^{2}(0, t ; H)}^{2}+\left\|\int_{0}^{t} \nabla \bar{w}\right\|_{H}^{2} \leq c \int_{0}^{t}\left(\gamma\left(w^{1}\right)-\gamma\left(w^{2}\right)-\bar{w}, \bar{\beta}_{1}\right) .
\end{aligned}
$$

Then, taking advantage of the Lipschitz continuity of $\gamma$ (cf. assumption (3.24) ) and using (3.29), we get

$$
\begin{aligned}
& \|\bar{p}\|_{L^{2}(0, t ; H)}^{2}+\left\|\int_{0}^{t} \nabla \bar{p}\right\|_{H}^{2}+\sum_{j=1}^{2}\left\|\bar{\beta}_{j}(t)\right\|_{H}^{2}+\nu \sum_{j=1}^{2}\left\|\nabla \bar{\beta}_{j}\right\|_{L^{2}(0, t ; H)}^{2} \\
& +\|\bar{w}\|_{L^{2}(0, t ; H)}^{2}+\left\|\int_{0}^{t} \nabla \bar{w}\right\|_{H}^{2} \leq \eta\|\bar{w}\|_{L^{2}(0, t ; H)}^{2}+c_{\eta} \int_{0}^{t}\left\|\bar{\beta}_{1}\right\|_{H}^{2},
\end{aligned}
$$

holding true for all positive constants $\eta$ and for some $c_{\eta}>0$. Finally, choosing $\eta$ sufficiently small and applying a standard version of the Gronwall lemma (cf. [10, Lemme A.2), we get the desired uniqueness of the $\boldsymbol{\beta}$ and $w$-components of the solution to Problem (P) and this concludes the proof of Theorem 3.10 .

5. Proof of Theorem 3.7. The following section is devoted to the proof of Theorem 3.7. It will be done by steps. First we approximate our Problem (P) by a more regular Problem $(\mathrm{P})^{\sigma}$, then (fixed $\sigma>0$ ) we find well-posedness for the approximating problem using a fixed-point theorem and then we perform some a priori estimates (independent of $\sigma$ ) on its solution. Finally we find the existence of solutions of Problem (P) by passing to the limit in Problem $(\mathrm{P})^{\sigma}$ as $\sigma \searrow 0$.

In order to perform the following estimates rigorously, we use here the Lipschitz continuous Yosida-Moreau approximation $\partial \varphi^{\sigma}=\left(\varphi^{\sigma}\right)^{\prime}$ (cf. [10, Prop. 2.11, p. 39]) of $\partial \varphi$.

5.1. The approximating problem. Let us introduce here the approximating Problem $(\mathrm{P})^{\sigma}$ of our Problem (P) (cf. equations (3.15)-(3.20) ). First of all, we take a small positive parameter $\sigma$ and we call $\gamma^{\sigma}$ the following Lipschitz continuous approximation of the function $\gamma(w)=\exp (w)$ in (3.7), i.e. the function

$$
\gamma^{\sigma}(w):= \begin{cases}\exp w & \text { if } r \leq 1 / \sigma \\ (w-1 / \sigma) \exp (1 / \sigma)+\exp (1 / \sigma) & \text { if } r \geq 1 / \sigma .\end{cases}
$$


Moreover let $\delta^{\sigma}$ be the inverse function of $\gamma^{\sigma}$, i.e.

$$
\delta^{\sigma}\left(\gamma^{\sigma}(r)\right)=r \quad \forall r \in \mathbb{R},
$$

and let $\widehat{\gamma}^{\sigma}$ be a primitive of the function $\gamma^{\sigma}$, i.e.

$$
\widehat{\gamma}^{\sigma}(r)=1+\int_{0}^{r} \gamma^{\sigma}(s) d s \quad \forall r \in \mathbb{R} .
$$

Then the following properties of $\gamma^{\sigma}$ hold true (cf. also [6, Lemma 5.1]).

LEMma 5.1. It follows that

$$
\widehat{\gamma}^{\sigma}(r) \geq \gamma^{\sigma}(r), \quad r\left(\delta^{\sigma}\right)^{\prime}(r) \geq 1 \quad \forall r \in \mathbb{R} .
$$

Now, we can approximate Problem (P) as follows.

Problem $(\mathrm{P})^{\sigma}$. Find $\left(w^{\sigma}, \beta_{1}^{\sigma}, \beta_{2}^{\sigma}\right)$ and $\left(\vartheta^{\sigma}, \boldsymbol{\xi}^{\sigma}, p^{\sigma}\right)$ with the following regularity properties:

$$
\begin{aligned}
& w^{\sigma}, \vartheta^{\sigma} \in H^{1}\left(0, T ; V^{\prime}\right) \cap L^{2}(0, T ; V), \\
& \beta_{1}^{\sigma}, \beta_{2}^{\sigma} \in H^{1}(0, T ; H), \quad \nu \beta_{1}^{\sigma}, \nu \beta_{2}^{\sigma} \in L^{\infty}(0, T ; V) \cap L^{2}(0, T ; W), \\
& \boldsymbol{\xi}^{\sigma} \in\left(L^{2}(0, T ; H)\right)^{2}, \quad p^{\sigma} \in H^{1}(0, T ; H) \cap L^{\infty}(0, T ; V) \cap L^{2}(0, T ; W),
\end{aligned}
$$

satisfying

$$
\begin{aligned}
& \partial_{t} p^{\sigma}+\varrho_{1} \partial_{t} \beta_{1}^{\sigma}+\varrho_{2} \partial_{t} \beta_{2}^{\sigma}+\mathcal{B} p^{\sigma}=0 \quad \text { a.e. in } Q_{T} \\
& \partial_{t} w^{\sigma}+\partial_{t} \beta_{1}^{\sigma}+\mathcal{B} w^{\sigma}=\mathcal{R} \quad \text { in } V^{\prime} \text { and a.e. in }[0, T] \\
& \partial_{t} \boldsymbol{\beta}^{\sigma}+\nu\left(\begin{array}{c}
\mathcal{B} \beta_{1}{ }^{\sigma} \\
\mathcal{B} \beta_{2}{ }^{\sigma}
\end{array}\right)+\boldsymbol{\xi}^{\sigma}-p^{\sigma}\left(\begin{array}{c}
\varrho_{1} \\
\varrho_{2}
\end{array}\right)=\left(\begin{array}{c}
\vartheta^{\sigma}-\vartheta_{c} \\
0
\end{array}\right) \quad \text { a.e. in } Q_{T}, \\
& \vartheta^{\sigma}=\gamma^{\sigma}\left(w^{\sigma}\right), \quad \boldsymbol{\xi}^{\sigma} \in \partial \varphi^{\sigma}\left(\boldsymbol{\beta}^{\sigma}\right), \quad \text { a.e. in } Q_{T},
\end{aligned}
$$

and such that

$$
\begin{aligned}
& w^{\sigma}(0)=w_{0}, \quad p^{\sigma}(0)=p_{0} \quad \text { a.e. in } \Omega, \\
& \boldsymbol{\beta}^{\sigma}(0)=\left(\beta_{1}^{\sigma}(0), \beta_{2}^{\sigma}(0)\right)=\boldsymbol{\beta}_{0} \quad \text { a.e. in } \Omega .
\end{aligned}
$$

Existence and uniqueness of solutions to Problem $(\mathrm{P})^{\sigma}$ directly follow from Theorem 3.10 . We proceed now by performing a priori estimates on this solution uniform in $\sigma$ in order to pass to the limit in Problem $(\mathrm{P})^{\sigma}$ as $\sigma \searrow 0$, recovering a solution to Problem (P).

5.2. A priori estimates. In this subsection we perform a priori estimates on Problem $(\mathrm{P})^{\sigma}$ uniformly in $\sigma$, which will lead us to pass to the limit in Problem $(\mathrm{P})^{\sigma}$ as $\sigma \searrow 0$ and recover a solution of PROBLEM $(\mathrm{P})$. Hence, let us denote by $c$ all the positive constants (which may also differ from line to line) independent of $\sigma$ and depending on the data of the problem.

Now, let us come to the (uniform in $\sigma$ ) estimates on the solution to Problem $(\mathrm{P})^{\sigma}$. 
First estimate. Test (5.7) by $p^{\sigma}$, (5.8) by $\vartheta^{\sigma}=\gamma^{\sigma}\left(w^{\sigma}\right)$, and (5.9) by $\partial_{t} \boldsymbol{\beta}^{\sigma}$. Then, sum up the result of the first two tests with the sum of the two components of the vectorial equation found by the third test. This, after an integration in time on $(0, t)$, with $t \in[0, T]$, thanks to a cancellation of two integrals, leads to

$$
\begin{aligned}
& \frac{1}{2}\left\|p^{\sigma}(t)\right\|_{H}^{2}+\left\|\nabla p^{\sigma}\right\|_{L^{2}(0, t ; H)}^{2}+\int_{\Omega} \widehat{\gamma}^{\sigma}\left(w^{\sigma}(t)\right)+\int_{0}^{t} \int_{\Omega} \nabla \delta^{\sigma}\left(\vartheta^{\sigma}\right) \nabla \vartheta^{\sigma} \\
& +\sum_{j=1}^{2}\left\|\partial_{t} \beta_{j}^{\sigma}\right\|_{L^{2}(0, t ; H)}^{2}+\frac{\nu}{2} \sum_{j=1}^{2}\left\|\nabla \beta_{j}^{\sigma}(t)\right\|_{H}^{2}+\int_{\Omega} \varphi^{\sigma}\left(\boldsymbol{\beta}^{\sigma}(t)\right) \leq \frac{1}{2}\left\|p_{0}\right\|_{H}^{2}+\int_{\Omega} \widehat{\gamma}^{\sigma}\left(w_{0}\right) \\
& +\int_{0}^{t}\left\langle\mathcal{R}, \vartheta^{\sigma}\right\rangle+\frac{\nu}{2} \sum_{j=1}^{2}\left\|\nabla \beta_{j}^{\sigma}(0)\right\|_{H}^{2}+\int_{\Omega} \varphi^{\sigma}\left(\boldsymbol{\beta}_{0}\right)+\int_{0}^{t} \int_{\Omega} \vartheta_{c} \partial_{t} \beta_{1}^{\sigma} .
\end{aligned}
$$

Now, following the line of [6, (5.5))-(5.7), p. 1583], we can deal with the source term $\mathcal{R}$, recalling (3.11) and using a well-known compactness inequality (cf. [19, Theorem 16.4]) in the following way:

$$
\begin{aligned}
\int_{0}^{t} \int_{\Omega} R \vartheta^{\sigma} & \leq \int_{0}^{t}\|R(s)\|_{L^{\infty}(\Omega)}\left\|\vartheta^{\sigma}(s)\right\|_{L^{1}(\Omega)} d s \\
\int_{0}^{t} \int_{\partial \Omega} \Pi \vartheta^{\sigma} & \leq c\|\Pi\|_{L^{\infty}(\Sigma)}\left\|\left(\vartheta^{\sigma}\right)^{1 / 2}\right\|_{L^{2}(\Sigma)} \\
& \leq \zeta\left\|\nabla\left(\vartheta^{\sigma}\right)^{1 / 2}\right\|_{L^{2}(0, t ; H)}^{2}+C_{\zeta}\left\|\left(\vartheta^{\sigma}\right)^{1 / 2}\right\|_{L^{2}(0, t ; H)}^{2}
\end{aligned}
$$

for all $\zeta>0$ and for some positive $c_{\zeta}$ depending also on $\|\Pi\|_{L^{\infty}(\Omega)}$ (cf. (3.10)) and on $\Omega$. Now, collecting estimates (5.14) (5.15), with $\zeta=1$, and using Lemma 5.1] with assumptions (3.8)-(3.10), we get the inequality:

$$
\begin{aligned}
& \frac{1}{2}\left\|p^{\sigma}(t)\right\|_{H}^{2}+\left\|\nabla p^{\sigma}\right\|_{L^{2}(0, t ; H)}^{2}+2 \int_{\Omega} \vartheta^{\sigma}(t)+\int_{0}^{t} \int_{\Omega} \frac{\left|\nabla \vartheta^{\sigma}(s)\right|^{2}}{\vartheta^{\sigma}(s)} d s \\
& +\frac{1}{2} \sum_{j=1}^{2}\left\|\partial_{t} \beta_{j}^{\sigma}\right\|_{L^{2}(0, t ; H)}^{2}+\frac{\nu}{2} \sum_{j=1}^{2}\left\|\nabla \beta_{j}^{\sigma}(t)\right\|_{H}^{2}+\int_{\Omega} \varphi^{\sigma}\left(\boldsymbol{\beta}^{\sigma}(t)\right) \\
& \leq c+\int_{0}^{t}\|R(s)\|_{L^{\infty}(\Omega)}\left\|\vartheta^{\sigma}(s)\right\|_{L^{1}(\Omega)} d s+c\left\|\left(\vartheta^{\sigma}\right)^{1 / 2}\right\|_{L^{2}(0, t ; H)}^{2} .
\end{aligned}
$$

Finally, a standard version of the Gronwall lemma gives:

$$
\begin{aligned}
& \left\|p^{\sigma}\right\|_{L^{\infty}(0, t ; H)}^{2}+\left\|\nabla p^{\sigma}\right\|_{L^{2}(0, t ; H)}^{2}+\left\|\left(\vartheta^{\sigma}\right)^{1 / 2}(t)\right\|_{H}^{2}+\int_{0}^{t}\left\|\nabla\left(\vartheta^{\sigma}\right)^{1 / 2}\right\|_{H}^{2} \\
& +\sum_{j=1}^{2}\left\|\partial_{t} \beta_{j}^{\sigma}\right\|_{L^{2}(0, t ; H)}^{2}+\sum_{j=1}^{2}\left\|\nabla \beta_{j}^{\sigma}(t)\right\|_{H}^{2}+\int_{\Omega} \varphi^{\sigma}\left(\boldsymbol{\beta}^{\sigma}(t)\right) \leq c .
\end{aligned}
$$

Second estimate. Testing (5.8) with $w^{\sigma}$ and using (5.16), it is a standard matter to deduce the following bound:

$$
\left\|w^{\sigma}\right\|_{L^{\infty}(0, T ; H) \cap L^{2}(0, T ; V)} \leq c,
$$

whence (5.8) entails

$$
\left\|w^{\sigma}\right\|_{H^{1}\left(0, T ; V^{\prime}\right)} \leq c
$$


while, recalling (2.22), from (5.16) we also get

$$
\left\|\vartheta^{\sigma}\right\|_{L^{5 / 3}\left(Q_{T}\right)} \leq c .
$$

Moreover, testing equation (4.6) by $\left|\boldsymbol{\xi}^{\sigma}\right|^{-1 / 3} \boldsymbol{\xi}^{\sigma}$, we get

$$
\left\|\xi_{i}^{\sigma}\right\|_{L^{5 / 3}\left(Q_{T}\right)} \leq c \quad(i=1,2),
$$

and so, by comparison in (5.9), we get

$$
\left\|\nu \beta_{i}^{\sigma}\right\|_{L^{5 / 3}\left(0, T ; W^{2,5 / 3}(\Omega)\right)} \leq c \quad(i=1,2) .
$$

Now it remains only to pass to the limit in (5.7)-(5.12) as $\sigma \searrow 0$. This will be the aim of the next subsection.

5.3. Passage to the limit. As we have just mentioned, we want to conclude the proof of Theorem 3.7 by passing to the limit in the well-posed (cf. Subsection 5.1) Problem (P) ${ }^{\sigma}$ as $\sigma \searrow 0$ using the previous uniform (in $\sigma$ ) estimates on its solution (cf. Subsection 5.2) and exploiting some compactness-monotonicity argument. First let us list the weak or weak-star convergence coming directly from the previous estimates and well-known weakcompactness results. Note that the following convergences hold only up to a subsequence of $\sigma \searrow 0$ (let us say $\sigma_{k} \searrow 0$ ). We denote it again with $\sigma$ only for simplicity of notation. From the estimates (5.16)-(5.21), we deduce that there exist $\left(w, \vartheta, \beta_{j}, p, \xi_{j}\right), j=1,2$, such that

$$
\begin{aligned}
w^{\sigma} \rightarrow w & \text { weakly star in } H^{1}\left(0, T ; V^{\prime}\right) \cap L^{2}(0, T ; V), \\
\vartheta^{\sigma} \rightarrow \vartheta & \text { weakly in } L^{5 / 3}\left(Q_{T}\right), \\
\beta_{j}^{\sigma} \rightarrow \beta_{j} & \text { weakly in } H^{1}(0, T ; H)(j=1,2), \\
\nu \beta_{j}^{\sigma} \rightarrow \nu \beta_{j} & \text { weakly star in } L^{\infty}(0, T ; V) \cap L^{5 / 3}\left(0, T ; W^{2,5 / 3}(\Omega)\right)(j=1,2), \\
p^{\sigma} \rightarrow p & \text { weakly star in } H^{1}(0, T ; H) \cap L^{\infty}(0, T ; V) \cap L^{2}(0, T ; W), \\
\xi_{j}^{\sigma} \rightarrow \xi_{j} & \text { weakly in } L^{5 / 3}\left(Q_{T}\right) \quad(j=1,2) .
\end{aligned}
$$

Moreover, employing the Aubin-Lions lemma (cf. [18, p. 58]) and [21, Cor. 8, p. 89], we also get:

$$
\begin{aligned}
w^{\sigma} \rightarrow w & \text { strongly in } L^{2}(0, T ; H), \quad \text { and hence a.e. in } Q_{T}, \\
\beta_{j}^{\sigma} \rightarrow \beta_{j} & \text { strongly in } C^{0}([0, T] ; H) \cap L^{2}(0, T ; V) \quad \text { if } \nu>0 \quad(j=1,2), \\
p^{\sigma} \rightarrow p & \text { strongly in } C^{0}([0, T] ; H) \cap L^{2}(0, T ; V) \quad \text { and hence a.e. in } Q_{T} .
\end{aligned}
$$

Note that (5.23), (5.28), and relation (5.10) imply immediately (cf. [18, Lemme 1.3, p. 12]) the convergence

$$
\vartheta^{\sigma} \rightarrow \vartheta=\gamma(w) \text { a.e. in } Q_{T},
$$

and by the Egorov theorem, we also deduce

$$
\vartheta^{\sigma} \rightarrow \vartheta \quad \text { strongly in } L^{q}\left(Q_{T}\right) \quad \forall q \in[1,5 / 3) .
$$

Finally, it remains to prove the identification of the maximal monotone graph $\partial \varphi$, i.e.

$$
\boldsymbol{\xi} \in \partial \varphi(\boldsymbol{\beta}) \text { a.e. in } Q_{T},
$$


with $\boldsymbol{\xi}=\left(\xi_{1}, \xi_{2}\right)$ and $\xi_{j}(j=1,2)$, which are the weak limits defined in (5.27). In order to do that we should verify that

$$
\limsup _{\sigma \searrow 0} \int_{0}^{T}\left(\boldsymbol{\xi}^{\sigma}, \boldsymbol{\beta}^{\sigma}\right) \leq \int_{0}^{T}(\boldsymbol{\xi}, \boldsymbol{\beta}) .
$$

The proof here is split into two parts.

Case $\nu>0$. In this case from (5.24)-(5.25), using again [21, Cor. 8, p. 89], we also deduce

$$
\beta_{j}^{\sigma} \rightarrow \beta_{j} \quad \text { strongly in } L^{r}\left(0, T ; L^{q}(\Omega)\right), \quad \forall r \in[1,+\infty), q \in[1,6)(j=1,2) .
$$

From this convergence and from (5.27) we immediately deduce that (5.32) is verified.

Case $\nu=0$. In case $\nu=0$, due to assumption (3.6), we also have that $\boldsymbol{\beta}$ is bounded in $L^{\infty}\left(Q_{T}\right)$. In this case we can prove that $\boldsymbol{\beta}^{\sigma}$ is a Cauchy sequence in $C^{0}([0, T] ; H)$. Indeed one can take the differences of equations (5.9) written for two different indices $\sigma$ and $\sigma^{\prime}$ and test it by the difference vector $\boldsymbol{\beta}^{\sigma}-\boldsymbol{\beta}^{\sigma^{\prime}}$. Take the differences of the two equations (5.7) integrated in time and test it by $p^{\sigma}-p^{\sigma^{\prime}}$. Summing up the two resulting equations and integrating over $(0, t), t \in(0, T)$, we get (as $\left.\sigma, \sigma^{\prime} \searrow 0\right)$ :

$$
\begin{aligned}
\left\|p^{\sigma}-p^{\sigma^{\prime}}\right\|_{L^{2}(0, t ; H)}^{2}+\frac{1}{2}\left\|\left(\boldsymbol{\beta}^{\sigma}-\boldsymbol{\beta}^{\sigma^{\prime}}\right)(t)\right\|_{H}^{2} & \leq \int_{Q_{t}}\left(\vartheta^{\sigma}-\vartheta^{\sigma^{\prime}}\right)\left(\beta_{1}^{\sigma}-\beta_{1}^{\sigma^{\prime}}\right) \\
& \leq 2\left\|\vartheta^{\sigma}-\vartheta^{\sigma^{\prime}}\right\|_{L^{1}\left(Q_{t}\right)} \rightarrow 0 .
\end{aligned}
$$

Hence, we have that $\boldsymbol{\beta}^{\sigma} \rightarrow \boldsymbol{\beta}$ strongly in $C^{0}([0, T] ; H)$ and weakly star in $L^{\infty}\left(Q_{T}\right)$ and so also strongly in $L^{r}\left(Q_{T}\right)$ for all $r \in[1,+\infty)$, which is sufficient in order to prove that (5.32) is satisfied.

Hence, in both cases, all these convergences with the identifications made above enable us to pass to the limit (as $\sigma \searrow 0$ or at least for a subsequence of it) in Problem (P) ${ }^{\sigma}$ finding a solution to Problem (P) and concluding in this way the proof of Theorem 3.7 . Note that the convergences hold only for proper subsequences $\sigma_{k}$ of $\sigma$ tending to 0 because of a lack of uniqueness of solutions (cf. also Remark 3.8). This concludes the proof of Theorem 3.7

\section{REFERENCES}

[1] Baiocchi C.: Sulle equazioni differenziali astratte lineari del primo e del secondo ordine negli spazi di Hilbert, Ann. Mat. Pura Appl. (4), 76, 233)-304 (1967). MR0223697 (36:6745)

[2] Barbu V.: Nonlinear Semigroups and Differential Equations in Banach Spaces, Noordhoff International Publishing, Leyden (1976). MR0390843 (52:11666)

[3] Bonetti E.: Modelling phase transitions via an entropy equation: Long-time behaviour of the solutions, Dissipative phase transitions, 21-42, Ser. Adv. Math. Appl. Sci., 71, World Sci. Publ., Hackensack, NJ (2006). MR2223371 (2006m:35361)

[4] Bonetti E., Colli P., Fabrizio M., Gilardi G.: Global solution to a singular integro-differential system related to the entropy balance, Nonlinear Anal. 66, 1949-1979 (2007). MR2304972

[5] Bonetti E., Colli P., Fabrizio M., Gilardi G.: Modelling and long-time behaviour of an entropy balance and linear thermal memory model for phase transitions, Discrete Contin. Dyn. Syst. Ser. B. 6, 1001-1026 (2006). MR2224868(2007c:80006)

[6] Bonetti E., Colli P., Frémond M.: A phase field model with thermal memory governed by the entropy balance, Math. Models Methods Appl. Sci. 13, no. 11, 1565-1588 (2003). MR2024463 (2004k:80007)

[7] Bonetti E., Frémond M.: A phase transition model with the entropy balance, Math. Meth. Appl. Sci. 26, 539-556 (2003). MR1967321(2004a:80008) 
[8] Bonetti E., Frémond M., Rocca E.: A new dual approach for a class of phase transitions with memory: Existence and long-time behaviour of solutions, J. Math. Pures Appl. 88, 455-481 (2007). MR2369878

[9] Bonetti E., Rocca E.: Global existence and long-time time behaviour for a singular integrodifferential phase-field system, Commun. Pure Appl. Anal. 6, 367-387 (2007). MR.2289826

[10] Brezis H.: Opérateurs Maximaux Monotones et Semi-groupes de Contractions dans les Espaces de Hilbert, North-Holland Math. Studies 5 North-Holland, Amsterdam (1973). MR 0348562 (50:1060)

[11] Brokate M., Sprekels J.: Hysteresis and Phase Transitions, Appl. Math. Sci. 121, Springer, New York (1996). MR1411908 (97g:35127)

[12] Colli P., Gilardi G., Grasselli M., Schimperna G.: Global existence for the conserved phase field model with memory and quadratic nonlinearity, Port. Math. (N.S.) 58, no. 2, 159-170 (2001). MR 1836260 (2002g:35112)

[13] Colli P., Sprekels J.: Positivity of temperature in the general Frémond model for shape memory alloys, Contin. Mech. Thermodyn. 5, no. 4, 255-264 (1993). MR.1247344 (94j:73009)

[14] Darcy H.: Les fontaines publiques de la ville de Dijon, V. Dalmont Paris (1856).

[15] Frémond M.: Non-smooth thermomechanics, Springer-Verlag, Berlin (2002). MR1885252 (2003g:74004)

[16] Frémond M., Rocca E.: Well-posedness of a phase transition model with the possibility of voids, Math. Models Methods Appl. Sci. 16, no. 4, 559-586 (2006). MR2218214 (2007k:80007)

[17] Germain P.: Cours de mécanique des milieux continus, Masson, Paris (1973). MR036854 (51:4782)

[18] Lions J.L.: Quelques Méthodes de Résolution des Problèmes aux Limites non Linéaires, Dunod, Gauthier-Villars, Paris (1969). MR0259693 (41:4326)

[19] Lions J.L., Magenes E.: Non-Homogeneous Boundary Value Problems and Applications, Vol. I, Springer-Verlag, Berlin (1972). MR0350177 (50:2670)

[20] Moreau J.J.: Fonctionnelles convexes, Collège de France (1966) and Dipartimento di Ingegneria Civile Università di Roma Tor Vergata (2003).

[21] Simon J.: Compact sets in the space $L^{p}(0, T ; B)$, Ann. Mat. Pura Appl. (4), 146, 65-96 (1987). MR916688 (89c:46055)

[22] Visintin A.: Models of Phase Transitions, Progress in Nonlinear Differential Equations and their Applications 28, Birkhäuser, Boston (1996). MR1423808 (98a:80006) 